\title{
A BOUNDARY-VALUE PROBLEM FOR THE BIHARMONIC EQUATION AND THE ITERATED LAPLACIAN IN A 3D-DOMAIN WITH AN EDGE
}

\author{
S. A. Nazarov* and G. H. Sweers ${ }^{\dagger}$
}

UDC 517.946

Let $\Omega$ be a domain with piecewise smooth boundary. In general, it is impossible to obtain a generalized solution $u \in W_{2}^{2}(\Omega)$ of the equation $\Delta_{x}^{2} u=f$ with the boundary conditions $u=\Delta_{x} u=0$ by solving iteratively a system of two Poisson equations under homogeneous Dirichlet conditions. Such a system is obtained by setting $v=-\Delta u$. In the two-dimensional case, this fact is known as the Sapongyan paradox in the theory of simply supported polygonal plates. In the present paper, the three-dimensional problem is investigated for a domain with a smooth edge $\Gamma$. If the variable opening angle $\alpha \in C^{\infty}(\Gamma)$ is less than $\pi$ everywhere on the edge, then the boundary-value problem for the biharmonic equation is equivalent to the iterated Dirichlet problem, and its solution u inherits the positivity preserving property from these problems. In the case $\alpha \in(\pi, 2 \pi)$, the procedure of solving the two Dirichlet problems must be modified by permitting infinite-dimensional kernel and co-kernel of the operators and determining the solution $u \in W_{2}^{2}(\Omega)$ by inverting a certain integral operator on the contour $\Gamma$. If $\alpha(s) \in(3 \pi / 2,2 \pi)$ for a point $s \in \Gamma$, then there exists a nonnegative function $f \in L_{2}(\Omega)$ for which the solution $u$ changes sign inside the domain $\Omega$. In the case of crack ( $\alpha=2 \pi$ everywhere on $\Gamma)$, one needs to introduce a special scale of weighted function spaces. In this case, the positivity preserving property fails. In some geometrical situations, the problems on well-posedness for the boundary-value problem for the biharmonic equation and the positivity property remain open. Bibliography: 46 titles.

\section{Formulation of The PROBlem AND A PRELIMINARY DESCRIPtion OF THE RESUlts}

1.1. Domain and boundary-value problems. Let $\Omega \subset \mathbb{R}^{3}$ be a domain with compact closure $\bar{\Omega}$ and a two-dimensional boundary $\partial \Omega$ that is smooth everywhere except for an edge. The edge $\Gamma$ is assumed to be a smooth, simple, and closed contour in $\mathbb{R}^{3}$. At each point $s \in \Gamma \subset \partial \Omega$, there exist two planes $\Pi^{ \pm}(s)$ that are tangent to the two surfaces $\partial \Omega^{ \pm}$. These two smooth surfaces remain from the splitting of $\partial \Omega$ by $\Gamma$. Let $\alpha(s)$ denote the angle between the planes $\Pi^{+}(s)$ and $\Pi^{-}(s)$ measured from inside $\Omega$. If

$$
\alpha(s) \in(0, \pi), \quad s \in \Gamma
$$

we say that the domain $\Omega$ is convex (locally convex) near the edge; if

$$
\alpha(s) \in(\pi, 2 \pi], \quad s \in \Gamma
$$

the domain becomes non-convex. If the function $\alpha$ is strictly monotone near a point $s_{0} \in \Gamma$ with $\alpha\left(s_{0}\right)=\pi$, then the edge changes from locally convex to locally concave. In the case $\alpha=2 \pi$, we call $\Gamma$ the front of a crack.

In the domain $\Omega$, we consider the boundary-value problem for the biharmonic equation:

$$
\Delta_{x}^{2} u(x)=f(x), \quad x \in \Omega, \quad u(x)=\Delta_{x} u(x)=0, \quad x \in \partial \Omega \backslash \Gamma .
$$

In the notation $w=-\Delta_{x} u$ and $v=u$, problem (1.3) reduces to the iterated Dirichlet problem for the Poisson equation:

$$
\begin{aligned}
& -\Delta_{x} w(x)=f(x), x \in \Omega, \quad w(x)=0, x \in \partial \Omega \backslash \Gamma, \\
& -\Delta_{x} v(x)=w(x), x \in \Omega, \quad v(x)=0, x \in \partial \Omega \backslash \Gamma .
\end{aligned}
$$

Following [1], a generalized solution to problem (1.4) is understood as an element $w$ of the Sobolev class $\stackrel{\circ}{W}_{2}^{1}(\Omega)$ (the symbol ${ }^{\circ}$ indicates the Dirichlet condition in problem (1.4)) that satisfies the integral identity

$$
\left(\nabla_{x} w, \nabla_{x} \psi\right)_{\Omega}=(f, \psi)_{\Omega} \quad \text { for any } \psi \in \stackrel{\circ}{W}{ }_{2}^{1}(\Omega) .
$$

*Institute of Applied Mechanical Engineering RAN, e-mail: serna@snark.ipme.ru.

${ }^{\dagger}$ Delft Institute of Applied Mathematics EWI, Delft University of Technology, Delft, The Netherlands. e-mail: g.h.sweers@ewi.tudelft.nl.

Translated from Zapiski Nauchnykh Seminarov POMI, Vol. 336, 2006, pp. 153-198. Original article submitted January 30, 2005. 
Here $\nabla_{x}=\operatorname{grad}$ and $(,)_{\Omega}$ is either the inner product in the Lebesgue space $L_{2}(\Omega)$ or the extension of the product to duality between the spaces $W_{2}^{-1}(\Omega)=\stackrel{\circ}{W} \underset{2}{1}(\Omega)^{*}$ and $\stackrel{\circ}{W} \underset{2}{1}(\Omega)$. It is known [1] that a generalized solution exists for any right-hand side $f \in W_{2}^{-1}(\Omega)$ without any restrictions on the boundary $\partial \Omega$, and that the inequality

$$
\left\|w ; H^{1}(\Omega)\right\| \leq c\left\|f ; H^{-1}(\Omega)\right\|
$$

is valid. Since $\stackrel{\circ}{W}=1(\Omega) \subset L_{2}(\Omega) \subset W_{2}^{-1}(\Omega)$, the iterated Dirichlet problems $(1.4),(1.5)$ give a solution $v \in \stackrel{\circ}{W}_{2}^{1}(\Omega)$ to problem (1.3) for which the inclusion $\Delta_{x} v \in \stackrel{\circ}{W} \underset{2}{1}(\Omega)$ holds with the estimate

$$
\left\|v ; W_{2}^{1}(\Omega)\right\|+\left\|\Delta_{x} v ; W_{2}^{1}(\Omega)\right\| \leq c\left\|f ; W_{2}^{-1}(\Omega)\right\|
$$

We emphasize that, in general, the above-mentioned inclusion for $\Delta_{x} v$ does not guarantee that all second-order derivatives of the function $v$ belong to the space $L_{2}(\Omega)$.

A generalized solution of problem (1.1) is understood as an element $u$ of the space

$$
W_{2,0}^{2}(\Omega):=W_{2}^{2}(\Omega) \cap \stackrel{\circ}{W}{ }_{2}^{1}(\Omega)
$$

that satisfies the integral identity

$$
\left(\Delta_{x} u, \Delta_{x} \Psi\right)_{\Omega}=(f, \Psi)_{\Omega}, \quad \Psi \in W_{2,0}^{2}(\Omega)
$$

For an arbitrary piecewise-smooth boundary $\partial \Omega$, the positive quadratic form in the left-hand side of (1.9) is not positive-definite on the subspace $W_{2,0}^{2}(\Omega)$ of the Sobolev space $W_{2}^{2}(\Omega)$; therefore, the variational formulation (1.9) of problem (1.3) does not provide directly the existence of a generalized solution but provides only its uniqueness. The solution $v$ given by problems (1.4) and (1.5) does not possess the necessary smoothness. Nevertheless, we show that in both situations (1.1) and (1.2), it is possible to construct a solution $u \in W_{2,0}^{2}(\Omega)$ to problem (1.3) via solutions to the iterated Dirichlet problem. If the domain is locally convex near the edge $\Gamma$, then $u$ coincides with the generalized solution $v \in \stackrel{\circ}{W} \frac{1}{2}(\Omega)$ of problem $(1.5)$ whose smoothness can easily be lifted (see Sec. 2.2). If the domain is locally concave, the solution $u \in W_{2,0}^{2}(\Omega)$ exists but differs from the solution $v$; to get the former solution, it is necessary to modify the domains of operators for problems (1.4) and (1.5) (see Sec. 3.1). If local convexity changes to locally concavity along the edge, the problem of existence of a solution $u \in W_{2,0}^{2}(\Omega)$ to problem (1.9) is open.

1.2. Sapongyan paradox in theory of plates. If the domain $\Omega$ is a polygon in the plane $\mathbb{R}^{2}$, problem $(1.3)$ describes the bending of an isotropic plate with a simply supported edge (the boundary condition $\Delta_{x} u=0$ changes on a part of the boundary where the curvature is not zero, see $[2, \S 30])$. In this case, the problem of existence of a generalized solution $u \in W_{2,0}^{2}(\Omega)$ is clear, since via integration by parts, the quadratic form in the left-hand side of (1.9) turns into a positive-definite form (cf. [3] and [2, §30]).

In [4], O. M. Sapongyan observed that for a nonconvex polygon, the method of conformal mappings applied to functions with finite Dirichlet integral provides a solution to problem (1.3) that does not possess finite elastic energy, i.e., does not belong to the class $W_{2}^{2}(\Omega)$. He did not find an explanation of this fact, and called it a paradox (see [4]).

The reason for the Sapongyan paradox was discovered in [5] (see also [39, §5.7] and [7, §5.8]). Namely, sequential solving of problems $(1.4)$ and (1.5) in the energy class $\stackrel{\circ}{W} \underset{2}{1}(\Omega)$ does not always lead to an energy solution $u \in W_{2,0}^{2}(\Omega)$ of problem (1.3). A sufficient condition for the solutions $u$ and $v$ to coincide is convexity of the polygon $\Omega$. Necessary conditions are certain orthogonality conditions for the right-hand side $f$; the number of these conditions is equal to the number of "concave" vertices of the polygon. Moreover, a procedure was developed in [5] which allows one to express, in a general situation, the solution $u \in W_{2,0}^{2}(\Omega)$ of problem (1.3) via solutions $w \in L_{2}(\Omega)$ and $v \in \stackrel{\circ}{W} \frac{1}{2}(\Omega)$ of problems (1.4) and (1.5). Let us briefly describe this procedure, assuming for simplicity that there is precisely one vertex $\mathcal{O}$ of the polygon $\Omega$ with angle $\alpha>\pi$. In this case, according to [8] (see also [39, $\S 2.3$ and 5.7]), any solution of problem (1.4) in the class $L_{2}(\Omega)$ takes the form

$$
w(x)=w_{0}(x)+a \zeta(x)
$$


where $w_{0} \in \stackrel{\circ}{W}_{2}^{1}(\Omega)$ is the (unique) generalized solution to the problem, $a$ is an arbitrary constant, and $\zeta$ is a harmonic function that vanishes on the polygonal line $\partial \Omega \backslash \mathcal{O}$ and admits the decomposition

$$
\zeta(x)=r^{-\pi / \alpha} \sin \left(\frac{\pi}{\alpha} \varphi\right)+O\left(r^{\pi / \alpha}\right) \quad \text { as } \quad r \rightarrow+0 .
$$

Here $(r, \varphi)$ are polar coordinates centered at $\mathcal{O}$, while the sides of the polygon $\Omega$ that go out from the vertex $\mathcal{O}$ are given by the equalities $\varphi=0$ and $\varphi=\alpha$. We emphasize that the function $\zeta$ exists for any angle $\alpha$, but only in the case $\alpha \in(\pi, 2 \pi]$ this function belongs to the space $L_{2}(\Omega)$.

According to [9] (see also [39, §2.3]), the solution $v \in \stackrel{\circ}{W}_{2}^{1}(\Omega)$ of problem (1.5) with right-hand side (1.10) lives in the space $W_{2,0}^{2}(\Omega)$, and thus becomes an energy solution of problem (1.3) if and only if the following orthogonality condition is satisfied:

$$
\int_{\Omega} w(x) \zeta(x) d x=0 .
$$

From (1.12) it follows that the constant $a$ in representation (1.10) must equal

$$
a=-\left\|\zeta ; L_{2}(\Omega)\right\|^{-2} \int_{\Omega} w_{0}(x) \zeta(x) d x .
$$

Thus, for a nonconvex polygon, problem (1.4) has to be solved in a wider class, while problem (1.3) has to be solved in a more narrow class than the intrinsic energy class $\stackrel{\circ}{W}_{2}^{1}(\Omega)$. The freedom appearing in the first step is canceled by the necessity to fulfill the compatibility conditions in the second step.

In a three-dimensional domain that is concave near the edge, analogous modifications are needed when we reduce the boundary-value problem for the biharmonic equation to the iterated Dirichlet problem for the Poisson equation. At the same time, the reduction procedure becomes much more complicated: namely, in formula (1.10), the term $a \zeta$ is replaced by an arbitrary element of a certain infinite-dimensional subspace $\operatorname{ker} \mathcal{A}_{2}^{1}$. Instead of the single condition (1.12), an infinite family of conditions of orthogonality to elements of the same subspace appears, and the algebraic equation (1.13) which eliminates the freedom in the choice of a solution to problem (1.4) becomes an integral equation on the contour $\Gamma$.

1.3. Infinite-dimensional kernels and co-kernels of operators for the Dirichlet Poisson problem. If $f \in L_{2}(\Omega)$, then there exists a unique solution $w$ to problem (1.4) in Kondrat'ev's weighted space $V_{1,0}^{2}(\Omega) \subset$ $\stackrel{\circ}{W}{ }_{2}^{1}(\Omega)$ (for the definition of this space and other function spaces, see Sec. 2.1). The above-mentioned reduction procedure forces us to consider two more continuous mappings:

$$
\stackrel{\circ}{\mathcal{A}}_{2}^{1}: V_{2,0}^{2}(\Omega) \rightarrow V_{2}^{0}(\Omega) \subset L_{2}(\Omega) \quad \text { and } \quad \stackrel{\circ}{\mathcal{A}_{0}^{1}}: V_{0,0}^{2}(\Omega) \rightarrow V_{0}^{0}(\Omega)=L_{2}(\Omega) .
$$

According to results of [10] (cf. Theorem 2.1 below), the operators in (1.11) are Fredholm, and even isomorphisms, in the case of a locally convex domain $\Omega$ only. If the domain is locally concave and, in addition,

$$
\alpha(s) \in(\pi, 2 \pi), \quad s \in \Gamma
$$

then $\stackrel{\circ}{\mathcal{A}}_{2}^{1}$ is an epimorphism with an infinite-dimensional kernel and $\stackrel{\circ}{\mathcal{A}}_{0}^{1}$ a monomorphism with an infinitedimensional co-kernel (cf. Theorem 2.3 and Corollary 2.1). Moreover, the operator $\stackrel{\circ}{\mathcal{A}}_{2}^{1}$ is formally adjoint to $\stackrel{\circ}{\mathcal{A}}_{0}^{1}$. If we consider these operators as unbounded operators in $L_{2}(\Omega)$ with domains given by formula (1.11), then $\stackrel{\circ}{\mathcal{A}}_{2}^{1}$ becomes a symmetric closed operator, while $\mathcal{\circ}_{0}^{1}$ is its adjoint operator $\left(\stackrel{\circ}{\mathcal{A}}_{0}^{1}\right)^{*}$. The latter facts can be checked with the help of Lemma 2.2 proven in Sec. 2 .

For a locally concave domain, the key problem is a description of the infinite-dimensional subspaces $\operatorname{ker} \mathcal{A}_{2}^{1} \simeq$ coker $\stackrel{\circ}{A}_{0}^{1}$. We solve this problem in Secs. 2.5 and 2.6 based on results of $[11-13]$ (see also [39, $\left.\S 9.4\right]$ ). In the sections mentioned, an intrinsic isomorphism is established between the subspace $\operatorname{ker}^{\circ} \mathcal{A}_{2}^{1}$ and the $\operatorname{space} H^{-\varkappa(\cdot)}(\Gamma)=$ $H^{\varkappa(\cdot)}(\Gamma)^{*}$, the dual for the Sobolev space $H^{\varkappa(\cdot)}(\Gamma)$ with a variable smoothness index $\varkappa(s)=1-\pi / \alpha(s)$. This 
index belongs to the interval $(0,1)$ due to condition (1.12). Such an interpretation of the kernel and co-kernel of operators (1.14) allows us to realize the above-described procedure of reducing problem (1.3) to problems (1.4), (1.5) under condition (1.12). The main role in the procedure is played by Theorem 3.1, which states that the freedom in determining the solutions $w \in V_{2,0}^{2}(\Omega) \subset L_{2}(\Omega)$ for problem (1.4) is large enough, but, at the same time, this freedom is completely exhausted by satisfying the compatibility conditions for problem (1.5) in the class $V_{0,0}^{2}(\Omega) \subset W_{2,0}^{2}(\Omega)$.

Restriction (1.12) is essential. Indeed, if $\alpha\left(s_{0}\right)=2 \pi$ at a point $s_{0} \in \Gamma$, then the operator of problem (1.3) with the domain $V_{\sigma}^{l+2}(\Omega)$ is not Fredholm for any weight index $\sigma \in C^{\infty}(\Gamma)$ (see Sec. 4.2). In Sec. 4.1, a body with a crack is examined, i.e.,

$$
\alpha(s)=2 \pi, \quad s \in \Gamma .
$$

In particular, an appropriate change of weighted norms for the function spaces (namely, the norms become "weight-stepped" [14, 15]) provides the Fredholm property of the operator for problem (1.3). In the last parts of Sec. 4, we discuss other geometrical situations that are not covered by the general scheme of Sec. 3 .

1.4. Positivity of solutions. The following property of solutions to problem (1.3) expresses the strict maximum principle:

$$
f \in L_{2}(\Omega) \backslash\{0\}, \quad f \geq 0 \Rightarrow w(x)>0, \quad x \in \Omega .
$$

If the domain is locally convex, then the generalized solution $u \in W_{2,0}^{2}(\Omega)$ of problem (1.9) obtained by consecutive solving problems (1.4) and (1.5) inherits, of course, the same property:

$$
f \in L_{2}(\Omega) \backslash\{0\}, \quad f \geq 0 \Rightarrow u(x)>0, \quad x \in \Omega .
$$

For a locally concave domain $\Omega$, problem (1.3) also possesses a positive solution $v \in \stackrel{\circ}{W}{ }_{2}^{1}(\Omega)$ with negative Laplacian $\Delta_{x} v \in \stackrel{\circ}{W} \underset{2}{1}(\Omega)$; however, the inclusion $v \in W_{2}^{2}(\Omega)$ does not necessarily hold. The change in the procedure of solving the Dirichlet problems (1.4) and (1.5) which leads to the solution $u \in W_{2,0}^{2}(\Omega)$ of problem (1.3) makes the validity of statements (1.16) doubtful. Indeed, in Secs. 3.4 and 4.2 we verify that, under condition (1.12) and the additional requirement that

$$
\text { there exists } s_{0} \in \Gamma \text { such that } \alpha\left(s_{0}\right)>3 \pi / 2 \text {, }
$$

or in the case (1.13) of a crack, one can find a nonnegative right-hand side $f \in L_{2}(\Omega)$ of problem (1.3) for which the solution $u \in W_{2,0}^{2}(\Omega)$ changes sign inside the domain $\Omega$. This example is based on the analysis of asymptotic behavior of the solution $u$ near the edge (Theorem 3.4 and Proposition 4.2).

The question whether the positivity preserving property (1.14) holds in the case

$$
\alpha(s) \in(\pi, 3 \pi / 2), \quad s \in \Gamma
$$

remains open. Numerical experiments predict a negative answer.

In the very beginning of the last century, T. Boggio and J. Hadamard conjectured that the solution $u \in \stackrel{\circ}{W}_{2}^{2}(\Omega)$ of the Dirichlet problem which describes bending of a plate with a clamped edge,

$$
\Delta_{x}^{2} u(x)=f(x), x \in \Omega, \quad u(x)=\partial_{n} u(x)=0, \quad x \in \partial \Omega \backslash \Gamma,
$$

possesses property (1.14). The motivation of Boggio (see [16]) was based on his results (see [17]) on positivity of the Green function for the polyharmonic operator $\left(-\Delta_{x}\right)^{m}$ in the ball $\mathbb{B} \in \mathbb{R}^{N}$. Hadamard wrote in [18] that there is no doubt that the conjecture holds for convex nice domains. He also was aware that for an annulus with a small interior hole, the hypotheses is wrong. Starting with the paper [19] published in 1949, a lot of counterexamples to the conjecture had appeared (cf. [20-28] etc). For instance, in [28] (see also [21]) it was shown that the Green function of problem (1.16) changes sign if $\Omega \in \mathbb{R}^{2}$ is an ellipse with axes ratio 2 . In [25], the sign change was established for rectangles (including squares). Thus, neither smoothness, nor uniform convexity, not even a rich symmetry of the domain do provide property (1.14) for problem (1.16). Furthermore, its first eigenfunction can change sign inside $\Omega$ (see [26, 27] and the review [29]).

Concerning domains for which the positivity preserving property holds, a result of [30] states that this property is preserved after adding lower-order operators with small coefficients to $\Delta^{2}$. This result allowed to establish 
property (1.14) in the case of some small convex perturbations of a circle (see [31]). Nonconvex domains for which solutions of problem (1.16) are subject to (1.14) were indicated by Hadamard in [19]. Indeed, he focused on Limaçons de Pascal (conformal images under $z \mapsto z+a z^{2}$ of the unit disk with $a \in\left[0, \frac{1}{2}\right]$ ). His statement is wrong: in [32], it was proven that property (1.14) holds for Limaçons de Pascal only in the case $a \in\left[0, \frac{1}{6} \sqrt{6}\right.$. However, a Limaçon de Pascal becomes a concave domain for $a>\frac{1}{4}$; therefore, nonconvexity is not sufficient for (1.14) to fail.

With the help of asymptotic methods (see [33] and $[7, \S 5.6]$ ), examples of solutions with variable sign to the Dirichlet problem (1.16) in a two-dimensional domain $\Omega$ can be adapted for long $(T \gg 1)$ cylinders $\Omega \times(-T, T) \in$ $\mathbb{R}^{3}$; moreover, the ends of cylinders can be rounded in order to get a smooth boundary.

1.5. Structure of the paper and some notation. In the next section, we collect information about solvability and asymptotic behavior of solutions of the Dirichlet problem for the Poisson equation in a domain with a smooth edge. If we cannot give precise references to the existing literature, we reconstruct proofs (Lemma 2.2 and Remark 2.3). Section 3 is concerned with a boundary-value problem for the biharmonic equation; in particular, in this section we obtain, under condition (1.12), necessary and sufficient conditions of solvability of this equation in the Kondrat'ev spaces. Moreover, asymptotic properties of solutions are examined and lost of positivity by a solution is verified in the case where condition (1.15) holds. In Sec. 4, a body with a crack is considered, and other pathologic (from the point of view of the general theory) geometric situations are discussed.

If several formulas are supplied with the same reference number, then an additional subscript indicates the number of formula item which we mean; for example, $(2.8)_{1}$ and $(2.8)_{2}$ refer to estimates of $w$ and $v$, respectively.

The notation $\stackrel{\circ}{W}_{2}^{1}(\Omega)$ and $W_{2,0}^{2}(\Omega)$ for subspaces where the variational statements of problems are formulated is taken from [1]. The Sobolev classes $H^{\varkappa(\cdot)}(\Gamma)$ with varying smoothness index $\varkappa$ and Kondrat'ev's weighted spaces $V_{\beta}^{l}(\Omega)$ have the same notation as in the monographs [46] and [6,35], respectively. As usual, by $H_{\text {loc }}^{l}(\Xi)$ we understand the linear space of functions whose restriction to any compact set $K \subset \Xi$ belongs to the Sobolev space $W_{2}^{l}(K)$.

\section{Solvability And asymptotics of solutions to the Dirichlet Poisson Problem}

2.1. Function spaces. Let $\rho \in C^{\infty}(\bar{\Omega} \backslash \Gamma)$ be a positive function that is equivalent to the $\operatorname{distance} \operatorname{dist}(x, \Gamma)$ inside a three-dimensional neighborhood $\mathcal{U}_{d}$ of the edge $\Gamma$. The Kondrat'ev space $V_{\beta}^{l}(\Omega)$ is defined as the completion of the lineal $C_{c}^{\infty}(\bar{\Omega} \backslash \Gamma)$ of infinitely differentiable functions that vanish near the contour $\Gamma$ with respect to the weighted norm:

$$
\left\|W ; V_{\beta}^{l}(\Omega)\right\|=\left(\sum_{k=0}^{l}\left\|\rho^{\beta-l+k} \nabla_{x}^{k} W ; L_{2}(\Omega)\right\|^{2}\right)^{1 / 2} .
$$

Here $\nabla_{x}^{k} U$ is the set of all derivatives of order $k$ of a function $U, l \in \mathbb{N}_{0}=\{0,1, \ldots\}$, and $\beta \in C^{\infty}(\bar{\Omega})$. If functions $\beta^{1}$ and $\beta^{2}$ coincide on the set $\Gamma$, then the corresponding spaces $V_{\beta^{1}}^{l}(\Omega)$ and $V_{\beta^{2}}^{l}(\Omega)$ can be distinguished neither algebraically nor topologically. Therefore, from now on we write $\beta \in C^{\infty}(\Gamma)$ without paying attention to the way of extension inside $\Omega$. The space $V_{\beta}^{l}(\Omega)$ is also invariant with respect to changes of the weight $\rho$ as long as the weight keeps its principal properties. This space consists of functions $W \in H_{\mathrm{loc}}^{l}(\bar{\Omega} \backslash \Gamma)$ with finite norm (2.1). The imbedding $V_{\beta}^{l}(\Omega) \subset V_{\gamma}^{m}(\Omega)$ holds under the conditions $l \geq m$ and $\beta-l \leq \gamma-m$, but this imbedding becomes compact only in the case of strict inequalities.

If $l \in \mathbb{N}=\{1,2, \ldots\}$, then the intrinsic norm of the trace space $V_{\beta}^{l-1 / 2}(\partial \Omega)$,

$$
\left\|w ; V_{\beta}^{l-1 / 2}(\partial \Omega)\right\|=\inf \left\{\left\|W ; V_{\beta}^{l}(\Omega)\right\| \mid W=w \text { on } \partial \Omega\right\}
$$

is equivalent to

$$
\left(\sum_{k=0}^{l-1}\left\|\rho^{\beta-l+k+1 / 2} \nabla_{\omega}^{k} w ; L_{2}(\partial \Omega)\right\|^{2}+\int_{\partial \Omega} \int_{\partial \Omega}\left|\rho(x)^{\beta(x)} \nabla_{\omega}^{l-1} w(x)-\rho(y)^{\beta(y)} \nabla_{\omega}^{l-1} w(y)\right|^{2} \frac{d \omega_{x} d \omega_{y}}{d(x, y)^{3}}\right)^{1 / 2} .
$$

Here $d \omega$ is an area element of the surface $\partial \Omega \backslash \Gamma, \nabla_{\omega}$ is the tangential gradient, and $d(x, y)$ is the distance between points $x, y \in \partial \Omega$ measured along the surface. In the case $\alpha(s)<2 \pi$, it is possible to replace $d(x, y)$ by the distance $|x-y|$ in $\mathbb{R}^{3}$ in formula (2.2), but in the case $\alpha\left(s_{0}\right)=2 \pi$, the functions $d(x, y)$ and $|x-y|$ are not equivalent since the surfaces of the "crack" may touch each other or even coincide as geometrical objects. 
Lemma 2.1. (1) The spaces $\stackrel{\circ}{W}_{2}^{1}(\Omega)$ and $\stackrel{\circ}{V_{0}^{1}}(\Omega)=\left\{v \in V_{0}^{1}(\Omega): v=0\right.$ on $\left.\partial \Omega\right\}$ coincide.

(2) The subspace $V_{\beta, 0}^{2}(\Omega)=V_{\beta}^{2}(\Omega) \cap \stackrel{\circ}{V}{ }_{\beta-1}^{1}(\Omega)$ is imbedded into $W_{2,0}^{2}(\Omega)$ if and only if $\beta(s) \leq 0$ for $s \in \Gamma$.

Proof. The first assertion was verified, for example, in [39, §2.3], while the second one follows from the definition of norm in (2.1).

Let $s$ be the arc length on the contour $\Gamma$, which is identified with a circle of a proper radius; with a certain ambiguity, we do not distinguish in notation a point on $\Gamma$ and its coordinate. The Sobolev-Slobodetskii space $H^{\varkappa(\cdot)}(\Gamma)$ with variable smoothness index

$$
\varkappa \in C^{\infty}(\Gamma), \quad 0<\varkappa(s)<1
$$

is equipped with the norm

$$
\left\|z ; H^{\varkappa(\cdot)}(\Gamma)\right\|=\left(\left\|z ; L_{2}(\Gamma)\right\|^{2}+\int_{0}^{h_{0}}\left\|h^{-\varkappa(\cdot)-1 / 2} \triangle_{h} z ; L_{2}(\Gamma)\right\|^{2} d h\right)^{1 / 2},
$$

where $h_{0}$ is a fixed positive number and $\triangle_{h} z(s)=z(s+h)-z(s)$ is the first-order difference. A description of principal properties of the space $H^{\varkappa(\cdot)}(\Gamma)$ (in particular, reflexivity) can be found, for example, in the monograph [46] and the literature cited therein. Furthermore, we use the dual space $H^{-\varkappa(\cdot)}(\Gamma)=H^{\varkappa(\cdot)}(\Gamma)^{*}$ as well.

2.2. Solvability of the Dirichlet problem. With the boundary-value problem,

$$
-\Delta_{x} w(x)=f(x), \quad x \in \Omega, \quad w(x)=g(x), \quad x \in \partial \Omega \backslash \Gamma,
$$

we associate the mapping

$$
\mathcal{A}_{\beta}^{l}=\left\{-\Delta_{x},\left.\right|_{\partial \Omega \backslash \Gamma}\right\}: V_{\beta}^{l+1}(\Omega) \rightarrow V_{\beta}^{l-1}(\Omega) \times V_{\beta}^{l+1 / 2}(\partial \Omega),
$$

which is continuous for any indices $l \in \mathbb{N}$ and $\beta \in C^{\infty}(\Gamma)$.

The following assertion is proved in [10] (see also [9] and $[39, \S 6.4]$ ).

Theorem 2.1. Let $l \in \mathbb{N}$. Mapping (2.5) is an isomorphism if and only if for any $s \in \Gamma$, the following inequalities hold:

$$
-\pi / \alpha(s)<\beta(s)-l<\pi / \alpha(s) .
$$

If one of the inequalities in (2.6) is violated at a point $s_{0} \in \Gamma$, then the operator $\mathcal{A}_{\beta}^{l}$ loses the Fredholm property, and in the case $\beta\left(s_{0}\right)-l>\pi / \alpha\left(s_{0}\right)$ (in the case $\beta\left(s_{0}\right)-l<-\pi / \alpha(s)$ ), this operator gets an infinite-dimensional kernel (co-kernel, respectively).

We verify in Remark 2.3 the absence of the Fredholm property of the operator $\mathcal{A}_{\beta}^{l}$ in the case where relations (2.6) are violated at a single point of the edge; in contrast to the remaining assertions of Theorem 2.3 , a precise reference to a proof of this fact is not known to the authors.

If the domain $\Omega$ is locally convex near the edge, then Theorem 2.1 admits the indices

$$
l=1 \quad \text { and } \quad \beta(s)=0,
$$

which provide that $V_{\beta}^{l-1}(\Omega)=L_{2}(\Omega)$ and $V_{\beta}^{l+1}(\Omega) \subset W_{2}^{2}(\Omega)$ (see Lemma 2.1, (2)). Hence, the generalized solutions $w \in \stackrel{\circ}{W} \underset{2}{1}(\Omega)$ and $v \in \stackrel{\circ}{W} \frac{1}{2}(\Omega)$ of problems $(1.4)$ and $(1.5)$ belong to the space $W_{2,0}^{2}(\Omega)$, and the relations

$$
c\left\|f ; V_{0}^{0}(\Omega)\right\|=c\left\|f ; L_{2}(\Omega)\right\| \quad \text { and } \quad\left\|v ; W_{2}^{2}(\Omega)\right\| \leq c\left\|v ; V_{0}^{2}(\Omega)\right\| \leq c\left\|w ; V_{0}^{0}(\Omega)\right\| \leq c\left\|w ; V_{0}^{2}(\Omega)\right\|
$$

are valid, i.e., $u=v \in W_{2,0}^{2}(\Omega)$ is a generalized solution of problem (1.3), and the following inequality holds:

$$
\left\|u ; W_{2}^{2}(\Omega)\right\| \leq c\left\|f ; L_{2}(\Omega)\right\| .
$$

If local convexity is absent, then the above conclusion is wrong since indices (2.7) do not meet the first condition of (2.6). 
2.3. Estimates for solutions with any weight index. Theorem 2.1 contains a restriction on the difference $\beta-l$ of the weight and smoothness indices; however, the estimate

$$
\left\|w ; V_{\beta}^{l+1}(\Omega)\right\| \leq c\left(\left\|f ; V_{\beta}^{l-1}(\Omega)\right\|+\left\|g ; V_{\beta}^{l+1 / 2}(\partial \Omega)\right\|+\| w ; V_{\beta-l-1}^{0}(\Omega)\right)
$$

is valid with any $\beta \in C^{\infty}(\Gamma)$ and $l \in \mathbb{N}$ under the condition that

$$
w \in H_{\mathrm{loc}}^{l+1}(\bar{\Omega} \backslash \Gamma) \cap V_{\beta-l-1}^{0}(\Omega), \quad\{f, g\} \in V_{\beta}^{l-1}(\Omega) \times V_{\beta}^{l+1 / 2}(\partial \Omega) .
$$

Estimate (2.10) is known, but since we cannot give an exact reference to its proof in the case of an edge with variable angle (and also for the reader convenience), we reproduce a complete verification of (2.10).

If $d>0$ is small, then we can introduce curvilinear coordinates $(y, s)$ in the $d$-neighborhood $\mathcal{U}_{d}$ of the contour $\Gamma$. Here $s$ is the arc length on $\Gamma$ and $y=\left(y_{1}, y_{2}\right)$ are Cartesian coordinates on the plane $\Pi_{\perp}(s)$ that is perpendicular to $\Gamma$ at the point $s$. We also define polar coordinates $(r, \varphi)$ on this plane assuming that $y_{1}=r \cos \varphi$ and $y_{2}=r \sin \varphi$. The planes $\Pi^{ \pm}\left(s_{0}\right)$ compose a dihedral angle $\mathbb{D}\left(s_{0}\right)$ with opening $\alpha\left(s_{0}\right)$ (from four possible angles, we choose the angle that is situated from the side of $\Omega$ ):

$$
\mathbb{D}\left(s_{0}\right)=\left\{\xi^{0} \in \mathbb{R}^{3}: \rho^{0}>0, \vartheta^{0} \in\left(\varphi_{0}\left(s_{0}\right), \varphi_{0}\left(s_{0}\right)+\alpha\left(s_{0}\right)\right), \xi_{3}^{0} \in \mathbb{R}\right\} .
$$

Here $\left(\rho^{0}, \vartheta^{0}, \xi_{3}^{0}\right)$ are cylindrical coordinates, and $\xi^{0}=\left(\xi_{1}^{0}, \xi_{2}^{0}, \xi_{3}^{0}\right)$ are the corresponding Cartesian coordinates related to the point $s_{0}$. If $s=s_{0}$, then $y_{i}=\xi_{i}^{0}$ for $i=1,2, r=\rho^{0}$, and $\varphi=\vartheta^{0}$. The curvilinear coordinates $(y, s)$ can be fixed so that $\varphi_{0} \in C^{\infty}(\Gamma)$.

Since the function $g \in V_{\beta}^{l+1 / 2}(\partial \Omega)$ has an extension $w_{g} \in V_{\beta}^{l+1}(\Omega)$ subjected to the relation $\left\|w_{g} ; V_{\beta}^{l+1}(\Omega)\right\| \leq$ $2\left\|g ; V_{\beta}^{l+1 / 2}(\partial \Omega)\right\|$, it is sufficient to verify inequality (2.10) in the case $g=0$, i.e., to consider the Dirichlet problem (1.4) with the right-hand side $f+\Delta_{x} w_{g}$. Moreover, thanks to the following local estimate of a solution to the Dirichlet problem:

$$
\left\|w ; W_{2}^{l+1}\left(\Omega \backslash \mathcal{U}_{d / 4}\right)\right\| \leq c\left(\left\|\Delta_{x} w ; W_{2}^{l-1}\left(\Omega \backslash \mathcal{U}_{d / 8}\right)\right\|+\left\|w ; L_{2}\left(\Omega \backslash \mathcal{U}_{d / 8}\right)\right\|\right),
$$

we may assume that the support of the function $w$ belongs to the set $\bar{\Omega} \backslash \mathcal{U}_{d / 2}$.

Let $s_{0} \in \Gamma$, let $\delta>0$, and let $D_{i}=\left\{x \in \Omega \cap \mathcal{U}_{d i / 2}:\left|s-s_{0}\right|<\delta i\right\}$ be the set that is cut out of $\Omega \cap \mathcal{U}_{d i / 2}$ by the planes $\Pi_{\perp}\left(s_{0} \pm \delta i\right)$; here $i=1,2$. Let us introduce "distorted cylindrical" coordinates:

$$
\rho=r=|y|, \quad \vartheta=\alpha(s)^{-1} \alpha\left(s_{0}\right)\left(\varphi-\varphi_{0}(s)+\varphi_{0}\left(s_{0}\right)\right), \quad \xi_{3}=s-s_{0},
$$

and the corresponding "distorted Cartesian" coordinates $\xi=\left(\rho \cos \vartheta, \rho \sin \vartheta, \xi_{3}\right)$. Then the set $D_{i}$ transforms into the part

$$
\mathcal{D}_{i}=\left\{\xi \in \mathbb{R}^{3}:\left|\xi_{3}\right|<\delta i, \rho<d\right\}
$$

of the dihedral angle (2.12). Let us also introduce the functions

$$
W(\xi)=\rho^{\beta\left(s_{0}\right)-\beta(s)} w(x) \quad \text { and } \quad F(\xi)=\rho^{\beta\left(s_{0}\right)-\beta(s)} f(x),
$$

which, according to definitions (2.13) and (2.1), satisfy the following relations:

$$
\begin{gathered}
\left\|w ; V_{\beta}^{l+1}\left(D_{1}\right)\right\| \leq c\left\|W ; V_{\beta\left(s_{0}\right)}^{l+1}\left(\mathcal{D}_{1}\right)\right\|, \quad\left\|W ; V_{\beta\left(s_{0}\right)-l-1}^{0}\left(\mathcal{D}_{2}\right)\right\| \leq c\left\|w ; V_{\beta-l-1}^{0}\left(D_{2}\right)\right\|, \\
\text { and }\left\|F ; V_{\beta\left(s_{0}\right)}^{l-1}\left(\mathcal{D}_{2}\right)\right\| \leq c\left\|f ; V_{\beta}^{l-1}\left(D_{2}\right)\right\| .
\end{gathered}
$$

Functions (2.14) satisfy the identity

$$
-\Delta_{x} W(\xi)-\rho^{\beta\left(s_{0}\right)-\beta(s)}\left[\Delta_{x}, \rho^{-\beta\left(s_{0}\right)+\beta(s)}\right] W(\xi)=F(\xi), \quad \xi \in \mathcal{D}_{2},
$$

where $[A, B]=A B-B A$ stands for the commutator of operators $A$ and $B$. The differential operators in the left-hand side are written in the $\xi$-coordinates, namely,

$$
\mathcal{L}\left(\xi, \nabla_{\xi}\right) W(\xi)=F(\xi), \quad \xi \in \mathcal{D}_{2} .
$$


In the set $\mathcal{D}_{i}$, we separate cylindrical layers

$$
\Xi_{i, k}=\left\{\xi \in \mathcal{D}_{i}: 2^{-k-1} d<i^{-1} \rho<2^{-k} d\right\}, \quad k \in \mathbb{N} ;
$$

in layers, we separate distorted "beams" (yokes)

$$
\Xi_{i, k}^{j}=\left\{\xi \in \Xi_{i, k}:\left|\delta^{-1} s+1-\left(j+\frac{1}{2}\right) 2^{1-k}\right|<i 2^{1-k}\right\}, \quad j=0, \ldots, 2^{k}-1 ;
$$

finally, in beams, we separate "bricks"

$$
\Xi_{i, k}^{j, q}=\left\{\xi \in \Xi_{i, k}^{j}:\left|\varphi-\varphi_{0}\left(s_{0}\right)-\left(q+\frac{1}{2}\right) \alpha_{0}\left(s_{0}\right) 2^{-k}\right|<i \alpha_{0}\left(s_{0}\right) 2^{-k}\right\}, \quad q=0, \ldots, 2^{k}-1 .
$$

The sets $\Xi_{1, k}^{q, j}$ belong to the sets $\Xi_{2, k}^{q, j}$ with diameter $O\left(2^{-k}\right)$, and the union of their closures coincides with $\overline{\mathcal{D}_{1}}$. The change of coordinates

$$
\xi \mapsto \eta=2^{k}\left(\xi-\xi^{k, j, q}\right),
$$

where $\xi^{k, j, q}$ is the mass center of the body $\Xi_{1, k}^{q, j}$, transfers $\Xi_{i, k}^{q, j}$ to the set $\widehat{\Xi}_{i, k}^{q, j}$ of unit sizes, and Eq. (2.17) is transformed to the following equation:

$$
2^{-2 k} \mathcal{L}\left(2^{-k} \eta-\xi^{j, k, q}, 2^{k} \nabla_{\eta}\right) W\left(2^{-k} \eta-\xi^{j, k, q}\right)=2^{-2 k} F\left(2^{-k} \eta-\xi^{j, k, q}\right), \quad \eta \in \widehat{\Xi}_{2, k}^{q, j} .
$$

If one face of a brick $\Xi_{i, k}^{q, j}$ lies on the boundary of the wedge $\mathbb{D}\left(s_{0}\right)$, then the function $\eta \mapsto W\left(2^{-k} \eta-\xi^{j, k, q}\right)$ vanishes on the corresponding face of the inflated brick $\widehat{\Xi} \underset{i, k}{q, j}$. Thus, local estimates for the solution of the Dirichlet problem hold for Eq. (2.17):

$$
\| \eta \mapsto W(\xi) ; W_{2}^{l+1}\left(\begin{array}{c}
\widehat{\Xi}_{1, k}^{q, j} \\
)
\end{array} \|^{2} \leq c\left(2^{-4 k}\left\|\eta \mapsto F(\xi) ; W_{2}^{l-1}\left(\widehat{\Xi}_{2, k}^{q, j}\right)\right\|^{2}+\left\|\eta \mapsto W(\xi) ; L_{2}\left(\widehat{\Xi}_{2, k}^{q, j}\right)\right\|^{2}\right) .\right.
$$

The constant $c$ in inequality (2.21) can be taken the same for all $k \in \mathbb{N}$ and $j, q=0, \ldots, 2^{k}-1$. This fact needs an explanation. First of all, the "gap" between the surfaces $\partial \widehat{\Xi}_{2, k}^{q, j} \backslash \partial \mathbb{D}\left(s_{0}\right)$ and $\partial \widehat{\Xi}_{1, k}^{q, j} \backslash \partial \mathbb{D}\left(s_{0}\right)$ is larger than some positive value which depends on $d, \delta$, and $\alpha\left(s_{0}\right)$, but does not depend on the numbers $k, j$, and $q$. Secondly, for a small $\delta>0$, the powers of $r$ in formula (2.13) vary slightly in the interval $\left(s_{0}-\delta, s_{0}+\delta\right)$; hence, the Laplacian in the left-hand side of (2.16) is perturbed by a small operator. Finally, by definition (2.13), the change $x \mapsto \xi$ is singular, i.e., coefficients at the function $W$ and its first-order derivatives $\partial W / \partial \xi_{p}$ in the expression $\mathcal{L} W$ have singularities $O\left(\rho^{-2}\right)$ and $O\left(\rho^{-1}\right)$, respectively, but the further coordinate dilation $\xi \mapsto \eta$ leads to a common large parameter $2^{2 k}$, which is compensated by the factor $2^{-2 k}$ in Eq. (2.20).

Let us return back to coordinates $\xi$ and rewrite estimate (2.21) as follows:

$$
\sum_{p=0}^{l+1} 2^{-2 k p}\left\|\nabla_{\xi}^{p} W ; L_{2}\left(\Xi_{1, k}^{q, j}\right)\right\|^{2} \leq c\left(\sum_{p=0}^{l-1} 2^{-2 k(p+2)}\left\|\nabla_{\xi}^{p} F ; L_{2}\left(\Xi_{2, k}^{q, j}\right)\right\|^{2}+\left\|W ; L_{2}\left(\Xi_{2, k}^{q, j}\right)\right\|^{2}\right) .
$$

Multiplying this inequality by $2^{-2 k\left(\beta\left(s_{0}\right)-l-1\right)}$ and noting that, by definition, the relation $c \rho \leq 2^{-k} \leq C \rho$ is valid on the brick $\Xi_{2, k}^{q, j}$ with positive constants $c$ and $C$, we transform Sobolev norms into weighted ones. As a result, we deduce that

$$
\left\|W ; V_{\beta\left(s_{0}\right)}^{l+1}\left(\Xi_{1, k}^{q, j}\right)\right\|^{2} \leq c\left(\left\|F ; V_{\beta\left(s_{0}\right)}^{l-1}\left(\Xi_{2, k}^{q, j}\right)\right\|^{2}+\left\|W ; V_{\beta\left(s_{0}\right)-l-1}^{0}\left(\Xi_{2, k}^{q, j}\right)\right\|^{2}\right) .
$$

Let us sum up inequalities (2.22) for $k \in \mathbb{N}$ and $j, q=1, \ldots, 2^{k}-1$. Since the multiplicity of the covering of the set $\mathcal{D}_{1}$ by bricks $\Xi_{2, k}^{q, j}$ is finite, we take formula (2.15) into account and conclude that

$$
\left\|w ; V_{\beta}^{l+1}\left(D_{1}\right)\right\|^{2} \leq c\left(\left\|f ; V_{\beta}^{l-1}\left(D_{2}\right)\right\|^{2}+\left\|w ; V_{\beta-l-1}^{0}\left(D_{2}\right)\right\|^{2}\right) .
$$

In order to derive the necessary estimate (2.10) in the simplified situation $\left(g=0\right.$ and supp $\left.u \subset \bar{\Omega}_{\mathrm{\Omega}} \overline{\mathcal{U}}_{d / 2}\right)$, it suffices now to cover the contour $\Gamma$ by $\operatorname{arcs}\left(s_{j}-\delta, s_{j}+\delta\right), j=0, \ldots, J-1$, restore from them the sets $D_{1}\left(s_{j}\right)$ and $D_{2}\left(s_{j}\right)$ (according to the instructions before formula (2.13)), and sum up inequalities (2.23) obtained on the fragments $D_{i}\left(s_{j}\right)$ of the set $\Omega \cap \mathcal{U}_{d i / 2}$ that adjoin the contour $\Gamma$. 
Lemma 2.2. Let $w$ be a solution to problem (2.4) with right-hand side $\{f, g\}$ such that inclusions (2.10) are valid. Then $w \in V_{\beta}^{l+1}(\Omega)$, and inequality (2.11) holds.

2.4. Asymptotics of a solution to the Dirichlet problem near the edge. According to results of [36], asymptotic behavior of a solution to problem (2.4) as $r=|y| \rightarrow 0$ is described in terms of special solutions to the model problem in a planar angle

$$
\mathbb{K}(s)=\left\{y \in \mathbb{R}^{2}: r>0, \varphi \in \Upsilon(s):=\left(\varphi_{0}(s), \varphi_{0}(s)+\alpha(s)\right)\right\},
$$

i.e., on the normal cross-section of the dihedral angle (2.12). The above-mentioned model problem:

$$
-\Delta_{y} U(y)=0, \quad y \in \mathbb{K}(s), \quad U(y)=0, \quad y \in \partial \mathbb{K}(s) \backslash\{0\}
$$

has the following solutions:

$$
U^{ \pm k}(y, s)=r^{ \pm k \pi / \alpha(s)} \sin \left(\frac{k \pi}{\alpha(s)}\left(\varphi-\varphi_{0}(s)\right)\right), \quad k \in \mathbb{N}
$$

(such solutions are called power-law ones).

Let us assume that for a certain $l \in \mathbb{N}$, the function $\beta$ satisfies inequalities (2.6); assume also that a function $\gamma \in C^{\infty}(\Gamma)$ meets the conditions

$$
-2 \pi / \alpha(s)<\gamma(s)-l<-\pi / \alpha(s), \quad s \in \Gamma,
$$

and

$$
\beta(s)>\gamma(s) \geq \beta(s)-1, \quad s \in \Gamma .
$$

In the sequel, we always assume that the domain $\Omega$ is concave near the edge. Since $1>|\pi / \alpha(s)| \geq \frac{1}{2}$ due to (1.2), for any index $\gamma$ subjected to condition (2.11) one can find a weight index $\beta \in C^{\infty}(\Gamma)$ such that requirements (2.6) and (2.28) are fulfilled. Note that under condition (1.12), the following indices fit:

$$
l=1, \quad \beta(s)=1, \quad \gamma(s)=0
$$

In the case of a crack $\alpha=2 \pi$, indices (2.29) (accepted in Sec. 3) are now forbidden by the first inequality in (2.28). The crack will be considered separately in Sec. 4, where restriction (1.28) is not necessary because it is easy to fulfill conditions (2.6), (2.27), and (2.28) for $\beta$ and $\gamma$.

Proposition 2.1. Let $l \in \mathbb{N}$ and assume that the smooth functions $\beta$ and $\gamma$ satisfy relations (2.6), (2.27), and (2.28). Then the solution $w \in V_{\beta}^{l+1}(\Omega)$ of problem (2.4) with right-hand side

$$
\{f, g\} \in V_{\gamma}^{l-1}(\Omega) \times V_{\gamma}^{l+1 / 2}(\partial \Omega)
$$

admits the asymptotic representation

$$
w(x)=\chi(r) K(s) U^{+1}(y, s)+\widetilde{w}_{0}(x) .
$$

Here $\chi \in C^{\infty}(\mathbb{R})$ is a cut-off function, $\chi(r)=1$ for $r<r_{0}$ and $\chi(r)=0$ for $r>2 r_{0}$, where the radius $r_{0}$ is chosen so that the support of the cut-off function $r \mapsto \chi(r)$ lies inside the neighborhood $\mathcal{U}_{d}$ at which curvilinear coordinates $(y, s)$ are introduced; the intensity factor $K$ is a function from the space $H^{\varkappa(\cdot)}(\Gamma)$ with smoothness index

$$
\varkappa(s)=l-\gamma(s)-\pi / \alpha(s),
$$

which meets condition (2.3) according to formulas (2.27) and (1.2); $U^{+1}$ is the power-law solution (2.26) whose homogeneity exponent is positive and minimal; and $\widetilde{w}_{0}$ is the asymptotic remainder in the space $V_{\beta-l-1}^{0}(\Omega)$.

Proof. See Remark 2.1.

The solution $w \in V_{\beta}^{l+1}(\Omega)$ and the asymptotic term $\chi K U^{+1}$ in $(2.31)$ belong to the space $V_{\beta-l-1}^{0}(\Omega)$ but not to the space $V_{\gamma-l-1}^{0}(\Omega)$ (the only exception is the case where the intensity factor is trivial). In this sense, 
formula (2.31) provides asymptotics of the solution to problem (2.4). However, the smoothness of the function $K$ is limited by relation $(2.32)$, which means in particular that $\varkappa(s)<1$. Thus, $\widetilde{w}_{0}$ it not differentiable. In [37] (see also [39, §8.1], [38, 13], and [39, §9.31 for general boundary-value problems), it is shown that a different asymptotic remainder gets the necessary smoothness properties if the formula

$$
w(x)=\mathfrak{C}(K ; r, s) U^{+}(y, s)+\widetilde{w}(x)
$$

contains a special extension operator $\mathfrak{C}$ from the edge $\Gamma$ into the domain $\Omega$. This operator can be defined with the help of a partition of unity and the standard extension operator from the axis $\mathbb{R}$ to the half-plane $\mathbb{R}_{+}^{2}$ :

$$
\mathfrak{C}_{0}(k ; r, z)=\int_{\mathbb{R}} X\left(r^{-1} \tau\right) k(z-\tau) r^{-1} d \tau=\int_{\mathbb{R}} X(t) k(z-r t) d t .
$$

Here the kernel $X$ is a rapidly decaying function with integral 1, i.e.,

$$
\left(1+t^{2}\right)^{\sigma}\left|\partial_{t}^{j} X(t)\right| \leq c_{\sigma, j}, \quad \sigma \in \mathbb{R}, j \in \mathbb{N}_{0}, \quad \text { and } \quad \int_{\mathbb{R}} X(t) d t=1 .
$$

The last property provides the equality $\mathfrak{E}_{0}(k ; 0, z)=k(z)$. One can take for $X$ the Fourier transform of the cut-off function $\chi$ which was introduced in formula $(2.31)$.

Let $\left\{\chi_{\Gamma}^{i}\right\}$ and $\left\{\chi_{\Omega}^{i}\right\}$ be finite families of functions from $C^{\infty}(\Gamma)$ and $C_{c}^{\infty}\left(\bar{\Omega} \cap \mathcal{U}_{d}\right)$, respectively, such that $\chi_{\Gamma}^{i} \chi_{\Omega}^{i}=\chi_{\Gamma}^{i}, \sum \chi_{\Gamma}^{i}=1$ on $\Gamma$, and the supports of these cut-off functions have small lengths. Of course, one can choose the $\chi_{\Omega}^{i}$ that depend on the variables $r$ and $s$ only. The operator $\mathfrak{C}$ in formula (2.33) looks as follows:

$$
\mathfrak{E}(K ; r, s)=\sum_{i} \chi_{\Omega}^{i}(r, s) \mathfrak{E}_{0}\left(\chi_{\Gamma}^{i} K ; r, s\right)
$$

The following inequalities (see, for example, [39, Propositions 6.4.7 and 9.3.10]) express properties of the extension operator:

$$
\begin{gathered}
\left\|x \mapsto \mathfrak{C}(K ; r, s)-\chi(r) K(s) ; V_{-\varkappa-1}^{0}(\Omega)\right\| \leq c\left\|K ; H^{\varkappa(\cdot)}(\Gamma)\right\|, \\
\left\|\nabla_{x}^{j} \mathfrak{C}(K) ; V_{j-\varkappa-1}^{0}(\Omega)\right\| \leq c_{j}\left\|K ; H^{\varkappa(\cdot)}(\Gamma)\right\| \text { for any } j \in \mathbb{N}, \\
\left\|\mathfrak{C}(K) ; V_{\varepsilon-1}^{0}(\Omega)\right\| \leq c_{\varepsilon}\left\|K ; H^{\varkappa(\cdot)}(\Gamma)\right\| \text { for any } \varepsilon>0 .
\end{gathered}
$$

The smoothness exponent $\varkappa$ in (2.36) must satisfy condition (2.3).

Theorem 2.2. Under the hypotheses of Proposition 2.1, the asymptotic decomposition (2.33) holds, where $\mathfrak{C}$ is the extension operator (2.35). The intensity factor $K \in H^{\varkappa(\cdot)}(\Gamma)$ and remainder $\widetilde{w} \in V_{\beta}^{l+1}(\Omega)$ satisfy the inequality

$$
\left\|K ; H^{\varkappa(\cdot)}(\Gamma)\right\|+\left\|\widetilde{w} ; V_{\gamma}^{l+1}(\Omega)\right\| \leq c\left(\left\|f ; V_{\gamma}^{l-1}(\Omega)\right\|+\left\|g ; V_{\gamma}^{l+1 / 2}(\partial \Omega)\right\|\right),
$$

with smoothness index $\varkappa$ as in (2.32).

A proof is given in the paper [37] (see also [39, Theorem 8.1.4]).

Remark 2.1. By inequality $(2.36)_{1}$ and relation $(2.32)$, the inclusion $(\mathfrak{C}(K)-\chi K) U^{+1} \in V_{-\varkappa-1-\pi / \alpha}^{0}(\Omega)=$ $V_{\gamma-l-1}^{0}(\Omega)$ is valid; therefore, decomposition (2.21) follows from formula (2.33), and, moreover, the norm $\left\|\widetilde{w} ; V_{\gamma-l-1}(\Omega)\right\|$ of the asymptotic remainder $\widetilde{w}$ does not exceed the right-hand side in $(2.37)$.

2.5. Integral representations of intensity factors. Let us search for a solution to the homogeneous problem (2.4) in the form

$$
\zeta(H ; x)=\chi(r) H(s) U^{-1}(y ; s)+\widehat{\zeta}(H ; x)
$$

where $H \in C^{\infty}(\Gamma)$, and $\widehat{\zeta}(H ; \cdot)$ is the solution supplied by Theorem 2.1 for the problem

$$
\begin{aligned}
-\Delta_{x} \widehat{\zeta}(H ; x) & =\widehat{f}(H ; x):=\Delta_{x} \chi(r) H(s) U^{-1}(y ; s), \quad x \in \Omega, \\
\widehat{\zeta}(H ; x) & =\widehat{g}(H ; x):=-\chi(r) H(s) U^{-1}(y ; s), \quad x \in \partial \Omega \backslash \Gamma .
\end{aligned}
$$


Remark 2.2. Let us describe natural curvilinear coordinates in the neighborhood $\mathcal{U}_{d}$ (cf. [39]). Denoting by $\mathbf{t}(s), \mathbf{n}(s)$, and $\mathbf{b}(s)$ the tangent, normal, and binormal vectors for the contour $\Gamma$, we fix $\left(y_{1}, y_{2}\right)$ to be the Cartesian coordinate system with axes $\mathbf{n}(s)$ and $\mathbf{b}(s)$. Then the Laplacian takes the following form:

$$
\begin{aligned}
\Delta & =\mathcal{L}\left(y, s, \nabla_{y}, \partial_{s}\right)=\frac{1}{\sqrt{g}}\left\{\frac{\partial}{\partial y_{1}} \frac{\sqrt{g}}{g_{11}} \frac{\partial}{\partial y_{1}}+\frac{\partial}{\partial y_{2}} \frac{\sqrt{g}}{g_{22}} \frac{\partial}{\partial y_{2}}+\frac{\partial}{\partial s} \frac{\sqrt{g}}{g_{s s}} \frac{\partial}{\partial s}\right\} \\
& =g_{s s}^{-1 / 2}\left\{\frac{\partial}{\partial y_{1}} g_{s s}^{1 / 2} \frac{\partial}{\partial y_{1}}+\frac{\partial}{\partial y_{2}} g_{s s}^{1 / 2} \frac{\partial}{\partial y_{2}}+\frac{\partial}{\partial s} g_{s s}^{-1 / 2} \frac{\partial}{\partial s}\right\}
\end{aligned}
$$

where $g_{11}, g_{22}$, and $g_{s s}$ are components of the metric tensor, and $g=g_{11} g_{22} g_{s s}$, while

$$
g_{11}=|\mathbf{n}|=1, \quad g_{22}=|\mathbf{b}|=1, \quad \text { and } \quad g_{s s}=\left|\mathbf{t}+y_{1} \partial_{s} \mathbf{n}+y_{2} \partial_{s} \mathbf{b}\right| .
$$

Moreover, according to the Serret-Frenet formula,

$$
\partial_{s} \mathbf{n}(s)=-k(s) \mathbf{t}(s)+\tau(s) \mathbf{b}(s) \quad \text { and } \quad \partial_{s} \mathbf{b}(s)=-\tau(s) \mathbf{n}(s),
$$

where $k(s)$ and $\tau(s)$ are the curvature and twisting of the arc $\Gamma$; therefore, $g_{s}(y, s)=\left(1-y_{1} k(s)\right)^{2}+r^{2} \tau(s)$. Thus, operator $(2.40)$ can be split as follows:

$$
\mathcal{L}\left(y, s, \nabla_{s}, \partial_{s}\right)=\Delta_{y}+\mathcal{L}_{1}\left(s, \nabla_{y}\right)+\widetilde{\mathcal{L}}\left(y, s, \nabla_{y}, \partial_{s}\right)
$$

where

$$
\mathcal{L}_{1}\left(s, \nabla_{y}\right)=-k(s) \frac{\partial}{\partial y_{1}}
$$

and the differential operator

$$
\widetilde{\mathcal{L}}=\sum_{p=1}^{2}\left(\sum_{q=1}^{2} l_{p, q}^{2} \frac{\partial^{2}}{\partial y_{p} \partial y_{q}}+l_{p}^{1} \frac{\partial}{\partial y_{p}}+l_{p s}^{1} \frac{\partial^{2}}{\partial y_{p} \partial s}\right)+l_{s}^{0} \frac{\partial}{\partial s}+l_{33}^{0} \frac{\partial^{2}}{\partial s^{2}}
$$

has smooth coefficients subjected to the conditions

$$
l_{\ldots}^{m}(y, s)=O\left(r^{m}\right) \quad \text { as } \quad r \rightarrow+0, \quad m=0,1,2 .
$$

In other words, for any $\lambda \in \mathbb{R}$ and $\Phi \in C^{2}\left(\mathbb{S}^{1} \times \Gamma\right)$, the following relation holds:

$$
\widetilde{\mathcal{L}}\left(y, s, \nabla_{y}, \partial_{s}\right) r^{\lambda} \Phi(\varphi, s)=O\left(r^{\lambda-2}\right) .
$$

Since $U^{-1}(s, \cdot)$ is a power-law solution of the model problem $(2.25)$, formulas $(2.41)-(2.43)$ show that

$$
\left|\nabla_{x}^{k} \widehat{f}(H ; x)\right| \leq c_{k} r^{-k-1-\pi / \alpha(s)}
$$

Hence, $\widehat{f}(H ; \cdot) \in V_{\beta}^{l-1}(\Omega)$ if

$$
\beta(s)>l-1+\pi / \alpha(s), \quad s \in \Gamma \text {. }
$$

Furthermore,

$$
\sin \frac{\pi}{\alpha(s)}\left(\varphi-\varphi^{0}(s)\right)=O(r), \quad x \in \partial \Omega_{ \pm} \cap \mathcal{U}_{d}
$$

because the left-hand side of (2.45) vanishes on the rays $\left\{x \in \Pi_{\perp}(s): r>0, \varphi=\varphi_{0}(s)\right\}$ and $\left\{x \in \Pi_{\perp}(s)\right.$ : $\left.r>0, \varphi=\varphi_{0}(s)+\alpha(s)\right\}$ which are tangent to the parts $\partial \Omega_{ \pm}$of the boundary $\partial \Omega$. In virtue of relation (2.45), the inclusion $\widehat{g}(H ; \cdot) \in V_{\beta}^{l+1 / 2}(\partial \Omega)$ is valid under the same condition $(2.44)$ on the weight index $\beta$. Due to the inequality $\alpha(s) \leq \pi$, one can find a function $\beta \in C^{\infty}(\Gamma)$ such that both restrictions in (2.44) and (2.6) are satisfied. Hence, by Theorem 2.1 , there exists a unique solution $\widehat{\zeta}(H ; \cdot) \in V_{\beta}^{l+1}(\Omega)$ of problem $(2.39)$; consequently, $\zeta(H ; \cdot)$ becomes a solution to the homogeneous problem $(2.4)$. This solution is nontrivial since $\chi H U^{-1} \notin V_{\beta}^{l+1}(\Omega)$ due to the second inequality in (2.6). 
Proposition 2.3. Under the hypotheses of Proposition 2.1, the following formula holds:

$$
\int_{\Omega} f(x) \zeta(H ; x) d x-\int_{\partial \Omega)} g(x) \partial_{n} \zeta(H ; x) d \omega=\pi \int_{\Gamma} K(s) H(s) d s .
$$

Here $\partial_{n}$ is the derivative along the outward normal to the surfaces $\partial \Omega_{ \pm}, K \in H^{\varkappa(\cdot)}(\Gamma)$ is the intensity factor from decompositions (2.33) and (2.31) of the solution $w$ to problem $(2.4)$, and $H \in C^{\infty}(\Gamma)$ is the density on $\Gamma$ which generates the weight function (2.38).

Proof. It is easy to check that under conditions $(2.27), \zeta(H ; \cdot) \in V_{2 l-\gamma}^{l+1}(\Omega)$. Hence, $\zeta(H ; \cdot) \in V_{l-\gamma-1}^{0}(\Omega)$, $\partial_{n} \zeta(H ; \cdot) \in V_{l-\gamma+1 / 2}^{0}(\partial \Omega)$, and, moreover, $f \in V_{\gamma-l+1}^{0}(\Omega)$ and $g \in V_{\gamma-l-1 / 2}^{0}(\partial \Omega)$ due to inclusion (2.45). In other words, integrals in the left-hand side of (2.46) converge. The relation itself is well known and can easily be obtained with the help of the Green formula in the domain $\Omega_{\delta}=\{x \in \Omega: \operatorname{dist}(x, \Gamma)>\delta\}$ and the limit passage as $\delta \rightarrow+0$. Indeed, the integral over the narrow curvilinear strip $\partial \Omega_{\delta} \backslash \partial \Omega$ can be calculated according to the asymptotic decompositions (2.33) and (2.38) and has the left-hand side of (2.46) as the limit. Necessary calculations for the Poisson equation can be found in [40] or in [39, Lemma 11.4.1] and, for general elliptic problems, in $[12,13]$ or $[39, \S 9.4]$.

Remark 2.3. Let us demonstrate that the range of the operator $\mathcal{A}_{\beta}^{l}$ is not closed if $\beta\left(s_{0}\right)-l=\pi / \alpha\left(s_{0}\right)$, while relation (2.6) holds for $s \in \Gamma \backslash\left\{s_{0}\right\}$. We emphasize that the case $\beta\left(s_{0}\right)-l=-\pi / \alpha\left(s_{0}\right)$ is considered in the same way after passing to the operator $\mathcal{A}_{2 l-\beta}^{l}$, which is the formally adjoint to $\mathcal{A}_{\beta}^{l}$, and therefore, is Fredholm simultaneously with $\mathcal{A}_{\beta}^{l}$ (see formulas (2.52) and (2.53) below and cf. Remark 3.2).

We introduce cut-off functions $\chi_{0}(t)=\chi_{+}(t) \chi_{+}(-t)$ and $\chi_{N}(t)=\chi_{+}(t-2 N) \chi_{+}(N-t)$; here $N \in \mathbb{N}$, $\chi_{+} \in C^{\infty}(\mathbb{R}), \chi_{+}(t)=0$ for $t>1, \chi_{+}(t)=1$ for $t<1 / 2$, and $0 \leq \chi_{+}(t) \leq 1$ for $t \in(1 / 2,1)$. For $\mu \in(1 / 2,1)$ and a sufficiently large $N$, the smooth function

$$
u_{n}(x)=U^{-1}(y, s) \chi_{N}(|\log r|) \chi_{0}\left(\left(s-s_{0}\right)|\log r|^{\mu}\right)
$$

has support in the set $\overline{\mathcal{U}_{d}} \cap(\bar{\Omega} \backslash \Gamma)$. Furthermore, the function $\tau=2(\beta-l-\pi / \alpha)$, which is nonnegative and vanishes at the point $s_{0}$ only, satisfies the inequality

$$
|\tau(s)| \leq c\left(s-s_{0}\right)^{2} .
$$

Hence, if $\left|s-s_{0}\right| \leq|\log r|^{-\mu}$ and $\mu>1 / 2$, then

$$
\left|r^{\tau(s)}-1\right|=\left|e^{\tau(s) \log r}-1\right| \leq c_{0}|\log r|^{1-2 \mu}
$$

therefore,

$$
\begin{gathered}
\left\|u_{N} ; V_{\beta-l-1}^{0}(\Omega)\right\|^{2} \geq \int_{e^{-2 N}}^{e^{-N}} \int_{s_{0}-|\log r|^{-\mu} / 2}^{s_{0}+|\log r|^{-\mu} / 2} r^{\tau(s)} \frac{d r}{r} d s \geq \int_{e^{-2 N}}^{e^{-N}}|\log r|^{-\mu}\left(1-c_{0}|\log r|^{1-2 \mu}\right) \frac{d r}{r} \\
\geq \int_{N}^{2 N} \lambda^{-\mu}\left(1-c_{0} N^{1-2 \mu}\right) d \lambda=\frac{1}{1-\mu} N^{1-\mu}\left(2^{1-\mu}-1\right)\left(1-c_{0} N^{1-2 \mu}\right) \geq \frac{1}{1-\mu}\left(2^{-\mu}-\frac{1}{2}\right) N^{1-\mu},
\end{gathered}
$$

where $N \geq N_{0}$, and the number $N_{0} \in \mathbb{N}$ is chosen so that $c_{0} N^{1-2 \mu} \leq 1 / 2$.

Consider now the expressions $\Delta_{x} u$ and $\left.u\right|_{\partial \Omega \backslash \Gamma}$. The supports of derivatives of the cut-off functions $\chi_{N}(|\log r|)$ are situated inside the union of the sets $\mathcal{V}_{d}^{N, i}=\left\{x \in \overline{\mathcal{U}_{d}}: e^{i-1} \leq r e^{N(i+1)} \leq e^{i}\right\}, i=0,1$. Derivatives of the second cut-off function $\chi_{0}\left(\left(s-s_{0}\right)|\log r|^{\mu}\right)$, which is defined in the neighborhood $\mathcal{U}_{d}$, can be different from zero only on the set $\mathcal{W}_{d, \mu}=\left\{x \in \overline{\mathcal{U}_{d}}:|\log r|^{\mu}\left(s-s_{0}\right) \in[1 / 2,1]\right\}$; these derivatives admit the estimate

$$
\left|\nabla_{y}^{p} \partial_{s}^{q} \chi_{0}\left(\left(s-s_{0}\right)|\log r|^{\mu}\right)\right| \leq c_{p, q} r^{-p}|\log r|^{\mu q-1-\delta_{p, 0}}
$$


where $p, q \in \mathbb{N}_{0}, p+q>0$, and $\delta_{m, h}$ is the Kronecker symbol. According to Remark 2.2,

$$
\left|\nabla_{y}^{m} \partial_{s}^{h}\left(\Delta_{x}-\Delta_{y}\right) u_{N}\right| \leq c_{m, h} r^{-m-1-\pi / \alpha(s)}, \quad m, h \in \mathbb{N}_{0}
$$

Thus, in view of the nonnegativity of the function $\tau \in C^{\infty}(\Gamma)$,

$$
\left\|\left(\Delta_{x}-\Delta_{y}\right) u_{N} ; V_{\beta}^{l-1}(\Omega)\right\|^{2} \leq c \int_{e^{-2 N}}^{e^{-N}} \int_{s_{0}-|\log r|^{-\mu} / 2}^{s_{0}+|\log r|^{-\mu} / 2} r^{\tau(s)} r d r d s \leq c \int_{e^{-2 N}}^{e^{-N}} r|\log r|^{-\mu} d r \leq c N^{-\mu} e^{-2 N} .
$$

Finally, the function $\Delta_{y} u_{N}$ does not vanish only on the union of the sets $\mathcal{V}_{d, \mu}^{N, i}=\left\{x \in \mathcal{V}_{d}^{N, i}:|\log r|^{\mu}\left(s-s_{0}\right) \in\right.$ $[1 / 2,1]\}, i=0,1$, and $\mathcal{W}_{d, \mu}^{N}=\left\{x \in \mathcal{W}_{d, \mu}: e^{-2 N-1} \leq r \leq e^{-N+1}\right\}$. Hence,

$$
\left\|\Delta_{y} u_{N} ; V_{\beta}^{l-1}(\Omega)\right\|^{2} \leq c\left(\int_{\mathcal{V}_{d, \mu}^{N, 0} \cup \mathcal{V}_{d, \mu}^{N, 1}} r^{\tau(s)} r^{-2} d x+\int_{\mathcal{W}_{d, \mu}^{N}} r^{\tau(s)}|\log r|^{-2} r^{-2} d x\right) \leq c\left(N^{-\mu}-N^{-\mu-2}\right) .
$$

In virtue of relation (2.45), traces of the function $u_{N}$ on the surfaces $\partial \Omega_{ \pm}$get an additional decay rate due to the factors $O(r)$, i.e., the following inequality:

$$
\left\|u_{N} ; V_{\beta}^{l+1 / 2}(\partial \Omega)\right\|^{2} \leq\left\|u_{N} ; V_{\beta+1 / 2}^{l+1}(\partial \Omega)\right\|^{2} \leq c N^{-\mu} e^{-2 N},
$$

analogous to (2.48), is valid. Under the hypotheses of Theorem 2.1, mapping (2.5) is an isomorphism; hence, the general scheme of $[10,9]$ for investigation of boundary-value problems in domains with edges, which includes local rectifying of the edge $\Gamma$ and surfaces $\partial \Omega_{ \pm}$, freezing coefficients of differential operators, and passing to the two-dimensional problem in an angle with the help of the partial Fourier transform, establishes that if $\beta\left(s_{0}\right)-l=\pi / \alpha\left(s_{0}\right)$, then the operator $\mathcal{A}_{\beta}^{l}$ remains an isomorphism. Thus, if this operator is Fredholm, then the following estimate holds:

$$
\left\|u_{N} ; V_{\beta-l-1}^{0}(\Omega)\right\| \leq\left\|u_{N} ; V_{\beta}^{l+1}(\Omega)\right\| \leq c\left(\left\|\Delta_{x} u_{N} ; V_{\beta}^{l-1}(\Omega)\right\|+\left\|\left.u_{N}\right|_{\partial \Omega} ; V_{\beta}^{l+1 / 2}(\partial \Omega)\right\|\right),
$$

where the constant $c$ is independent of the function $u_{N} \in V_{\beta}^{l+1}(\Omega)$. Estimate (2.51) cannot be true due to the verified inequalities (2.47)-(2.50). Thus, even if restriction (2.7) is violated at a single point $s_{0} \in \Gamma$, then operator (2.5) of problem (1.3) loses the Fredholm property.

2.6. Description of the kernel and co-kernel of the Dirichlet problem without a unique solution. According to Theorem 2.1, the operator $\mathcal{A}_{\gamma}^{l}$ with weight index $\gamma$ that satises relation (2.27) is not Fredholm because this operator has an infinite-dimensional co-kernel. Indeed, by Theorem 2.2, problem (2.4) with right-hand side $(2.40)$ has a solution $w \in V_{\gamma}^{l+1}(\Omega)$ only if the following equalities hold:

$$
K(s)=0, \quad s \in \Gamma
$$

these equalities imply an infinite family of compatibility conditions.

By a closing argument, the formula

$$
\left(\Delta_{x} u, \eta\right)_{\Omega}+\left(u, \partial_{n} \eta\right)_{\partial \Omega}=\left(u, \Delta_{x} \eta\right)_{\Omega}+\left(\partial_{n} u, \eta\right)_{\partial \Omega}
$$

can be extended to couples of functions $u \in V_{\gamma}^{l+1}(\Omega)$ and $\eta \in V_{2 l-\gamma}^{l+1}(\Omega)$ without any restrictions on the weight index $\gamma$. Theorem 6.3.3 of [39] demonstrates that the operator $\mathcal{A}_{2 l-\gamma}^{l}$ is formally adjoint to $\mathcal{A}_{\gamma}^{l}$, and the subspace coker $\mathcal{A}_{\gamma}^{l}$ consists of the functionals

$$
\{f, g\} \mapsto \int_{\Omega} f(x) \zeta(x) d x-\int_{\partial \Omega)} g(x) \partial_{n} \zeta(x) d \omega
$$

which are generated by all elements $\zeta$ of the subspace ker $\mathcal{A}_{2 l-\gamma}^{l}$. Thus, the Green formula (2.52) and the related structure of the defect functionals (2.53) establish the natural isomorphism

$$
\operatorname{ker} \mathcal{A}_{2 l-\gamma}^{l} \approx \operatorname{coker} \mathcal{A}_{\gamma}^{l} \text {. }
$$

Let $\gamma$ and $\varkappa$ satisfy relations (2.27) and (2.32). For the density $H \in C^{\infty}(\Gamma)$, formula (2.30) defines an element of the subspace $\operatorname{ker} \mathcal{A}_{2 l-\gamma}^{l}$. In $[39, \S 9.4]$ the following fact is verified. 
Theorem 2.3. Assume that a sequence $\left\{H_{m}\right\}_{n=1}^{\infty}$ of smooth functions converges in $\left\|\cdot ; H^{-\varkappa(\cdot)}(\Gamma)\right\|$ to a distribution $H \in H^{-\varkappa(\cdot)}(\Gamma)$. Then the sequence $\left\{\zeta\left(H_{m} ; \cdot\right)\right\}_{n=1}^{\infty}$ of solutions (2.38) of the homogeneous problem (2.4) converges in the space $V_{2 l-\gamma}^{l+1}(\Omega)$ to a certain element of the kernel $\mathcal{A}_{2 l-\gamma}^{l}$ which is denoted by $\zeta(H ; \cdot)$. Any element of the subspace $\operatorname{ker} \mathcal{A}_{2 l-\gamma}^{l}$ can be obtained in this way. The inequalities

$$
c\left\|H ; H^{-\varkappa(\cdot)}(\Gamma)\right\| \leq\left\|\zeta(H ; \cdot) ; V_{2 l-\gamma}^{l+1}(\Omega)\right\| \leq C\left\|H ; H^{-\varkappa(\cdot)}(\Gamma)\right\|
$$

are valid with positive constants $c$ and $C$ which are independent of the distribution $H \in H^{-\varkappa(\cdot)}(\Gamma)$.

Thus, the mapping

$$
H^{-\varkappa(\cdot)}(\Gamma) \ni H \mapsto \zeta(H ; \cdot) \in V_{2 l-\gamma}^{l+1}(\Omega)
$$

defined originally by formula (2.38) for smooth functions $H$, is extended by completion to the isomorphism

$$
H^{-\varkappa(\cdot)}(\Gamma) \approx \operatorname{ker} \mathcal{A}_{2 l-\gamma}^{l}
$$

Relations (2.31) and (2.54) specified by formulas (2.56) and (2.53) present a description of the infinite-dimensional kernel of the operator $\mathcal{A}_{2 l-\gamma}^{l}$ and co-kernel of the operator $\mathcal{A}_{\gamma}^{l}$.

Corollary 2.1. Let $l \in \mathbb{N}$ and assume that the function $\gamma$ satisfies inequalities (2.27). Problem (2.4) with right-hand side (2.30) has a solution $w \in V_{\gamma}^{l+1}(\Omega)$ if and only if the conditions

$$
\int_{\Omega} f(x) \zeta(Z ; x) d x-\int_{\partial \Omega)} g(x) \partial_{n} \zeta(Z ; x) d \omega=0, \quad Z \in C^{\infty}(\Gamma),
$$

are met. By completion argument, equalities (2.58) remain true for any $Z \in H^{-\varkappa(\cdot)}(\Gamma)$, where $\varkappa$ is the function in (2.32).

2.7. Revisiting formula for the intensity factors. Under restriction (1.12), $\varkappa=l-\gamma-\pi / \alpha \in(0, \pi / \alpha)$ according to restriction (2.27); therefore, it is possible to choose the index $\gamma$ so that the condition $\varkappa>1 / 2$ is fulfilled on $\Gamma$. Then the subspace $H^{-\varkappa(\cdot)}(\Gamma)$ includes the Dirac $\delta$-function. Let $s_{0} \in \Gamma$ and let $\zeta^{M P}\left(s_{0} ; \cdot\right) \in$ ker $\mathcal{A}_{2 l-\gamma}^{l}$ be the image of the Dirac function $H(s)=\delta\left(s-s_{0}\right)$ determined by formulas (2.56) and (2.57). Equality (2.25) takes the following form:

$$
K\left(s_{0}\right)=\frac{1}{\pi}\left(\int_{\Omega} f(x) \zeta^{M P}\left(s_{0} ; x\right) d x-\int_{\partial \Omega} g(x) \partial_{n} \zeta^{M P}\left(s_{0} ; x\right) d \omega\right)
$$

this equality directly delivers the value of the intensity factor at the point $s_{0}$. The normalized solution $\pi^{-1} \zeta^{M P}\left(s_{0} ; \cdot\right)$ of the homogeneous problem (2.4) is called the Maz'ya-Plamenevskii weight function (see [41-43]).

If $H$ is a sufficiently smooth density, then, according to [40], the following relation holds:

$$
\zeta(H ; x)=\int_{\Gamma} H(s) \zeta^{M P}(s ; x) d s
$$

\section{Generalized solution to the Biharmonic operator,} ITS ASYMPTOTICS, AND THE POSITIVITY PRESERVING PROPERTY

3.1. Procedure of construction of a generalized solution. Let us adapt the algorithm of [5] (see also [39, $\S 6.3]$ and $[7, \S 5.8])$ to construct a solution of the two-dimensional problem (1.3) for the three-dimensional case assuming that the geometrical condition (1.12) is satisfied and fixing the smoothness and weight indices in (2.29). Namely, instead of the unique solution $w_{0} \in V_{1,0}^{2}(\Omega) \subset \stackrel{\circ}{W} \frac{1}{2}(\Omega)=\stackrel{\circ}{V_{0}^{1}}(\Omega)$ of problem (1.4) (see Theorem 2.1 and Lemma 2.1), we take the general solution of the problem:

$$
w(x)=w_{0}(x)+\zeta(H ; x)
$$


in a wider class $V_{2,0}^{2}(\Omega) \subset L_{2}(\Omega)$ (see Theorem 2.3 with $l=1$ and $\gamma=0$ ). The unknown density

$$
H \in H^{-\varkappa(\cdot)}(\Gamma), \quad \varkappa(s)=1-\pi / \alpha(s),
$$

is found in the process of solving problem (1.5) with right-hand side (3.1). Indeed, according to Corollary 2.1, the latter problem has a solution $v \in V_{2,0}^{2}(\Omega) \subset W_{2,0}^{2}(\Omega)$ if the compatibility conditions (2.58) are met. Due to formula (3.1), these conditions turn into an integral identity:

$$
\int_{\Omega} \zeta(H ; x) \zeta(Z ; x) d x=-\int_{\Omega} w_{0}(x) \zeta(Z ; x) d x, \quad Z \in C^{\infty}(\Gamma) .
$$

The left-hand side of (3.3) is the value at $Z$ of a continuous functional on the space $H^{-\varkappa(\cdot)}(\Gamma)$. By definition (2.1) and inequalities (2.55) and (1.6),

$$
\begin{gathered}
\left|\left(w_{0}, \zeta(Z ; \cdot)\right)_{\Omega}\right| \leq c\left\|w_{0} ; L_{2}(\Omega)\right\|\left\|\zeta(Z ; \cdot) ; L_{2}(\Omega)\right\| \\
\leq c\left\|w_{0} ; W_{2}^{1}(\Omega)\right\|\left\|\zeta(Z ; \cdot) ; V_{2}^{2}(\Omega)\right\| \leq c\left\|f ; L_{2}(\Omega)\right\|\left\|Z ; H^{-\varkappa(\cdot)}(\Gamma)\right\| .
\end{gathered}
$$

By the same reasoning, the left-hand side of $(3.3)$ can be represented as $(\mathcal{S} H, Z)_{\Gamma}$, where $(\cdot, \cdot)_{\Gamma}$ is an extension of the inner product in $L_{2}(\Gamma)$ to the duality between the spaces $H^{-\varkappa(\cdot)}(\Gamma)$ and $H^{\varkappa(\cdot)}(\Gamma)$, and the operator

$$
\mathcal{S}: H^{-\varkappa(\cdot)}(\Gamma) \rightarrow H^{\varkappa(\cdot)}(\Gamma)
$$

is continuous, symmetric, and positive. These properties are guaranteed by the definition

$$
(\mathcal{S} H, Z)_{\Gamma}=(\zeta(H ; \cdot), \zeta(Z ; \cdot))_{\Gamma}, \quad H, Z \in C^{\infty}(\Gamma),
$$

the inclusion $\zeta(Z ; \cdot) \in V_{2,0}^{2}(\Omega) \subset L_{2}(\Omega)$ for any density $Z \in H^{-\varkappa(\cdot)}(\Gamma)$, and the estimate

$$
\begin{gathered}
\left|(\zeta(H ; \cdot), \zeta(Z ; \cdot))_{\Omega}\right| \leq\left\|\zeta(H ; \cdot) ; L_{2}(\Omega)\right\|\left\|\zeta(Z ; \cdot) ; L_{2}(\Omega)\right\| \\
\leq\left\|\zeta(H ; \cdot) ; V_{2}^{2}(\Omega)\right\|\left\|\zeta(Z ; \cdot) ; V_{2}^{2}(\Omega)\right\| \leq c\left\|H ; H^{-\varkappa(\cdot)}(\Gamma)\right\|\left\|Z ; H^{-\varkappa(\cdot)}(\Gamma)\right\|,
\end{gathered}
$$

which is provided by $(2.55)$.

The first inequality in (2.55) and Lemma 2.2 with indices (2.29) make the operator $\mathcal{S}$ positive-definite:

$$
(\zeta(H ; \cdot), \zeta(H ; \cdot))_{\Omega}=\left\|\zeta(H ; \cdot) ; V_{0}^{0}(\Omega)\right\|^{2} \geq C\left\|\zeta(H ; \cdot) ; V_{2}^{2}(\Omega)\right\|^{2} \geq c\left\|H ; H^{-\varkappa(\cdot)}(\Gamma)\right\|^{2}, \quad c>0,
$$

and, hence, invertible. An explanation of (3.6) goes as follows: since $\zeta(H ; \cdot)$ is a solution to the homogeneous problem (2.4), the right-hand side of the applied estimate (2.10) loses the norms of the functions $f$ and $g$.

Theorem 3.1. If $f \in L_{2}(\Omega)$, then there exists a unique solution (3.2) of the integral identity (3.3), and

$$
\left\|H ; H^{-\varkappa(\cdot)}(\Gamma)\right\| \leq c\left\|f ; L_{2}(\Omega)\right\| .
$$

Proof. The verified properties of operator in (3.5) result in the existence and uniqueness of a solution, and estimate (3.7) follows from inequality (3.4).

The next assertion gives us a generalized solution of the boundary-value problem (1.3) for the biharmonic equation, i.e, a function $u \in W_{2,0}^{2}(\Omega)$ that satisfies the integral identity (1.7).

Theorem 3.2. Let the geometrical restriction (1.12) be met. If $f \in L_{2}(\Omega)$ and $H \in H^{-\varkappa(\cdot)}(\Gamma)$ is the density given by Theorem 3.1, then the solution $v \in \stackrel{\circ}{W} \underset{2}{1}(\Omega)$ of problem (1.5) with right-hand side (3.1) from the space $V_{2,0}^{2}(\Omega) \subset L_{2}(\Omega)$ falls into the space $V_{2}^{4}(\Omega) \cap V_{0,0}^{2}(\Omega) \subset W_{2}^{2}(\Omega)$, i.e., $u=v$ is a generalized solution of problem (1.3). The estimate

$$
\left\|v ; V_{2}^{4}(\Omega)\right\|+\left\|w ; V_{2}^{2}(\Omega)\right\| \leq c\left\|f ; L_{2}(\Omega)\right\|
$$

is valid, which establishes, in particular, inequality (2.9).

Proof. By Corollary 2.1, a solution $v \in V_{2}^{4}(\Omega) \cap V_{0,0}^{2}(\Omega)$ of problem (1.5) exists due to relation (3.3). According to formula (3.1), estimate (3.8) for the solution $w$ of problem (1.4) is guaranteed by Theorems 2.1, 2.3, and 3.1, while estimate in (3.8) for the obtained solution $v$ of problem (1.5) becomes true because under condition (2.27), which holds for indices $l=3$ and $\gamma=2$, the mapping

$$
\left\{-\Delta_{x},\left.\right|_{\partial \Omega \backslash \Gamma}\right\}: V_{\gamma}^{l+1}(\Omega) \rightarrow \operatorname{Im} \mathcal{A}_{\gamma}^{l}
$$

is an isomorphism (see $[39, \S 9.4])$. Finally, inequality $(2.9)$ follows from the imbedding $V_{2}^{4}(\Omega) \subset V_{0}^{2}(\Omega) \subset W_{2}^{2}(\Omega)$ (see Lemma 2.1, (2)). 
3.2. Solvability of the boundary-value problem for the biharmonic equation in weighted spaces. Problem (1.3) is naturally associated with the mapping

$$
V_{\sigma}^{l+2}(\Omega) \ni u \rightarrow \mathcal{B}_{\sigma}^{l} u=\left\{-\Delta_{x}^{2} u,\left.\Delta_{x} u\right|_{\partial \Omega \backslash \Gamma},\left.u\right|_{\partial \Omega \backslash \Gamma}\right\} \in \mathcal{R}_{\sigma}^{l} V(\Omega)=V_{\sigma}^{l-2}(\Omega) \times V_{\beta}^{l-1 / 2}(\partial \Omega) \times V_{\beta}^{l+3 / 2}(\partial \Omega),
$$

which is continuous for any $l \in \mathbb{N}_{2}=\{2,3, \ldots\}$ and $\sigma \in C^{\infty}(\Gamma)$. Let us detect for which indices the operator $\mathcal{B}_{\sigma}$ is an isomorphism.

If a solution $u \in V_{\sigma}^{l+2}(\Omega)$ of the boundary-value problem

$$
\Delta_{x}^{2} u(x)=f(x), \quad x \in \Omega, \quad \Delta_{x} u(x)=g_{1}(x), \quad u(x)=g_{0}(x), \quad x \in \partial \Omega \backslash \Gamma,
$$

with right-hand side

$$
\left\{f, g_{1}, g_{0}\right\} \in \mathcal{R}_{\sigma}^{l} V(\Omega)
$$

appears as a result of solving the Dirichlet problems

$$
-\Delta_{x} w(x)=f(x), x \in \Omega, \quad w(x)=-g_{1}(x), \quad x \in \partial \Omega \backslash \Gamma,
$$

and

$$
-\Delta_{x} v(x)=w(x), x \in \Omega, \quad v(x)=g_{0}(x), \quad x \in \partial \Omega \backslash \Gamma,
$$

in the spaces $V_{\sigma}^{l}(\Omega) \ni w$ and $V_{\sigma}^{l+2}(\Omega) \ni v$, respectively, then Theorem 2.1, with the changes $(l, \beta) \mapsto(l-1, \sigma)$ and $(l, \beta) \mapsto(l+1, \sigma)$, results in the conditions

$$
-\pi / \alpha(s)<\sigma(s)-(l-1)<\pi / \alpha(s), \quad-\pi / \alpha(s)<\sigma(s)-(l+1)<\pi / \alpha(s) .
$$

Assume that the domain $\Omega$ is locally convex (see condition (1.1)); then the interval in the following formula is not empty:

$$
\sigma(s)-l \in(1-\pi / \alpha(s),-1+\pi / \alpha(s)),
$$

which gives us the desired relations in (3.14). For such indices, a solution $u=v \in V_{\sigma}^{l+2}(\Omega)$ exists, and

$$
\left\|u ; V_{\sigma}^{l+2}(\Omega)\right\| \leq c\left\|\left\{f, g_{1}, g_{0}\right\} ; \mathcal{R}_{\sigma}^{l} V(\Omega)\right\| .
$$

If the domain is locally concave near the edge $\Gamma$ (cf. condition (1.2)), then it becomes impossible to satisfy the requirements of Theorem 2.1, and, by analogy with the preceding section, it is necessary to modify the procedure of solving problems (3.12) and (3.13).

Let condition (1.12) be satisfied and assume that indices $l$ and $\sigma$ are related by the formula

$$
|\sigma(s)-l| \leq \min \left\{1-\frac{\pi}{\alpha(s)}, \frac{2 \pi}{\alpha(s)}-1\right\} .
$$

According to Theorem 2.3, after the change $(l, 2 l-\gamma) \mapsto(l-1, \sigma)$, the general solution to problem (3.12) in the class $V_{\sigma}^{l}(\Omega)$ takes the form (3.1). Here $w_{0} \in V_{\beta-1}^{l}(\Omega)$ is the solution imposed by Theorem 2.1 with indices $l \geq 2$ and $\beta$ that meet conditions (2.6). The function $\zeta(H, \cdot)$ is a solution of the homogeneous problem with an arbitrary density $H \in H^{-\varkappa(\cdot)}(\Gamma)$. The smoothness index

$$
\varkappa(s)=l-\gamma(s)-\pi / \alpha(s)=\sigma(s)-l+1-\pi / \alpha(s)
$$

is computed according to formula (2.32), but takes into account the changes of indices. By relations (3.16) and (1.2), index (3.17) admits restriction (2.3). Note that all the restrictions on indices, which appear while applying assertions of Sec. 2, are ensured by inequality (3.16).

In view of Corollary 2.1, where $(l, \gamma) \mapsto(l+1, \sigma)$, the existence condition for a solution $v \in V_{\sigma}^{l+2}(\Omega)$ of problem (3.13) is precisely equality (2.58), where $f=w \in V_{\sigma}^{l}(\Omega)$ and $g=g_{0} \in V_{\sigma}^{l+3 / 2}(\partial \Omega)$. The latter equality turns into the integral identity

$$
\int_{\Omega} \zeta(H ; x) \zeta(Z ; x) d x=-\int_{\Omega} w_{0}(x) \zeta(Z ; x) d x-\int_{\partial \Omega)} g_{0}(x) \partial_{n} \zeta(Z ; x) d \omega, \quad Z \in H^{-\varkappa(\cdot)}(\Gamma) .
$$

The same reasoning as in the preceding section allows us to find the density $H$ and function $v=u \in V_{\sigma}^{t+2}(\Omega)$ which solve problems (3.18), (3.13), and (3.12), respectively. 
Theorem 3.3. Let $l \in \mathbb{N}_{2}$ and assume that condition (1.1) (condition (1.12)) is fulfilled. Operator (3.9) for problem (3.10) is an isomorphism if and only if the weight index $\sigma \in C^{\infty}(\Gamma)$ satisfies inclusion (3.15) (inequality (3.16), respectively). If the above-mentioned conditions are violated, then the operator $\mathcal{B}_{\sigma}^{l}$ loses the Fredholm property.

Proof. The proof will be completed in Remark 3.2.

Remark 3.1. If $\alpha\left(s_{0}\right)=2 \pi$, then the left-hand side of inequality (3.16) vanishes at the point $s_{0} \in \Gamma$, and one cannot obey the conditions in Theorem 3.3, i.e., mapping (3.9) is not Fredholm for any index $\sigma$ (cf. Remark 2).

3.3. Power-law solutions of the model problem for the biharmonic equation. As in the case of the Dirichlet problem (2.4), asymptotic behavior of solutions to the boundary-value problem (3.10) is described by power-logarithmic solutions of the corresponding model problem in the angle (2.24):

$$
-\Delta_{y}^{2} U(y)=0, y \in \mathbb{K}(s), \quad \Delta_{y} U(y)=0, U(y)=0, \quad y \in \partial \mathbb{K}(s) \backslash\{0\} .
$$

These solutions can be easily presented. If $\alpha(s) \in(0, \pi) \cup(\pi, 2 \pi)$, then any power-logarithmic solution of problem (3.19) becomes a linear combination of the following solutions:

$$
U^{k, j \pm}(y, s)=r^{2 j \pm k \pi / \alpha(s)} \sin \left(\frac{k \pi}{\alpha(s)}\left(\varphi-\varphi_{0}(s)\right)\right), \quad k \in \mathbb{N}, \quad j=0,1 .
$$

The functions $U^{k, 0 \pm}$ do not differ from the harmonic power-law solutions $(2.26)$. In the case $\alpha(s)=2 \pi$, two functions,

$$
U^{2,0+}(y, s)=U^{2,1-}(y, s)=r \sin \left(\varphi-\varphi_{0}(s)\right),
$$

from list (3.20) coincide, and one of them is replaced by the power-logarithmic solution

$$
U^{2,1 / 2}(y, s)=(4 \pi)^{-1} r \log r \sin \left(\varphi-\varphi_{0}(s)\right)
$$

Problem (3.19) does not have any other power-logarithmic solutions.

In the case $\alpha(s) \in(0, \pi)$, the set of homogeneity exponents $\lambda$ of functions (3.20) that satisfy the inequality $\operatorname{Re} \lambda \geq 1$ has the lower bound

$$
\lambda_{+}(\alpha(s))=\pi / \alpha(s)
$$

If $\alpha(s) \in(\pi, 2 \pi]$, the lower bound gets the following form:

$$
\lambda_{+}(\alpha(s))= \begin{cases}2-\pi / \alpha(s) & \text { for } \quad \alpha(s) \in(\pi, 3 \pi / 2] \\ 2 \pi / \alpha(s) & \text { for } \quad \alpha(s) \in[3 \pi / 2,2 \pi]\end{cases}
$$

Remark 3.2. In notation (3.23) and (3.24), conditions on the weight index $\sigma$ in Theorem 3.3 convert into

$$
l+1-\sigma(s) \in I(s)=\left(2-\lambda_{+}(\alpha(s)), \lambda_{+}(\alpha(s))\right) .
$$

Indices $\sigma$ and $2 l-\sigma$ satisfy inclusion (3.25) simultaneously; therefore, the existence of the inverse operators for $\mathcal{B}_{\sigma}^{l}$ and $\mathcal{B}_{2 l-\sigma}^{l}$ has been proved in the preceding section. By Theorem 6.3.3 of [39], the above operators are formally adjoint because the boundary-value problem (3.10) is formally self-adjoint. Hence, each of the operators is an isomorphism. Ends of the interval $I(s)$ are homogeneity exponents of nontrivial power-law solutions of the model problem (3.19); the interval itself is free of such exponents. It is known (see [9] and, for example, [39, $\S 6.3])$ that there may exist only one interval that possesses the above-mentioned properties and provides the Fredholm property of the operator $\mathcal{B}_{\sigma}^{l}$. The latter property is absent if $l+2-\sigma(s)-(3-1) / 2 \notin I(s)$ at a certain point $s_{0} \in \Gamma$ (the numbers 3 and 1 are the dimensions of the manifolds $\Omega$ and $\Gamma$ ).

Remark 3.3. Similarly to (3.25), condition (2.6) in Theorem 2.1 reads as follows:

$$
l-\beta(s)=l+1-\beta(s)-(3-1) / 2 \in I(s)=(-\pi / \alpha(s), \pi / \alpha(s)) .
$$

At the same time, ends of the interval in (3.26) are exponents of the power solutions (2.26) of the model problem (2.25) for $k=1$. 


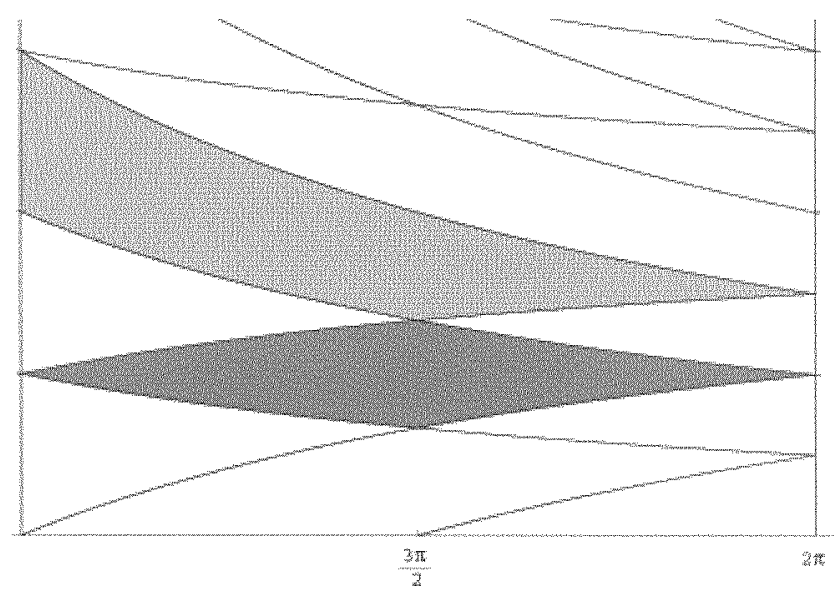

FIG. 1. Lines of forbidden indices and their dependence on $\alpha(s)$. The zone with dark shading is related to index $\sigma$.

\subsection{Asymptotics for solutions to the biharmonic equation.}

Assume that the domain $\Omega$ is concave near the edge and the edge $\Gamma$ does not turn into a crack front at any point $s$ (compare formulas (1.2) and (1.12)). Consider a solution $u \in V_{\sigma}^{l+2}(\Omega)$ of problem (3.10) with right-hand side

$$
\left\{f, g_{1}, g_{0}\right\} \in \mathcal{R}_{\gamma}^{l} V(\Omega),
$$

where index $\sigma$ meets the hypotheses of Theorem 3.3 and index $\gamma \in C^{\infty}(\Gamma)$ is chosen so that

$$
\sigma(s)>\gamma(s) \geq \sigma(s)-1 \quad \text { and } \quad \max \{2 \pi / \alpha(s), 2-\pi / \alpha(s)\}<l+1-\gamma(s)<3 \pi / \alpha(s)
$$

(cf. formulas (2.28), (3.20), and (3.25), respectively). Admissible ranges for the values $l+1-\sigma(s)$ and $l+1-\gamma(s)$ with their dependence on the angle $\alpha(s)$ are shown in Fig. 1.

Theorem 3.4. Under the above-formulated requirements on $\Omega,\left\{f, g_{1}, g_{0}\right\}, l, \sigma$, and $\gamma$, a solution $u \in V_{\sigma}^{l+2}(\Omega)$ of problem (3.10) admits the decomposition

$$
u(x)=\mathfrak{C}\left(K_{1} ; r, s\right) U^{1,1-}(y, s)+\mathfrak{C}\left(K_{2} ; r, s\right) U^{2,0+}(y, s)+\widetilde{u}(x) .
$$

Here $\mathfrak{C}$ is the extension operator (2.35) and the $K_{i}$ are intensity factors in the spaces $H^{\varkappa_{i}(\cdot)}(\Gamma)$ with smoothness exponents

$$
\varkappa_{1}(s)=l-1-\gamma(s)+\pi / \alpha(s) \quad \text { and } \quad \varkappa_{2}(s)=l+1-\gamma(s)-2 \pi / \alpha(s)
$$

which satisfy condition (2.3) due to assumptions (1.12), (3.16), and (3.27). The remainder $\widetilde{u}$ belongs to the space $V_{\gamma}^{l+2}(\Omega)$, and

$$
\sum_{i=1}^{2}\left\|K_{i} ; H^{\varkappa_{i}(\cdot)}(\Gamma)\right\|+\left\|\widetilde{u} ; V_{\gamma}^{l+1}(\Omega)\right\| \leq c\left\|\left\{f, g_{1}, g_{0}\right\} ; \mathcal{R} V_{\gamma}^{l}(\Omega)\right\|
$$

Proof. If either $\alpha(s) \in(\pi, 3 \pi / 2)$ or $\alpha(s) \in(3 \pi / 2,2 \pi)$ for $s \in \Gamma$, then formulas (3.28)-(3.30) follow directly from results of $[13]$ (see also $[39, \S 11.3]$ ). In the general case $\alpha(s) \in(\pi, 2 \pi)$, the graphs of the functions

$$
\Gamma \ni s \mapsto 2-\pi / \alpha(s) \quad \text { and } \quad \Gamma \ni s \mapsto 2 \pi / \alpha(s)
$$

intersect, which, in principle, is forbidden in the above-cited publications. However, proofs of the corresponding assertions can be repeated without any modifications for the problem under consideration even in the case of intersecting graphs (3.31) on some parts of the edge $\Gamma$. The point is that the power-law solutions $U^{1,1-}$ and $U^{2,0+}$ in the asymptotic formula (3.31) do not "interact" (cf. Remark 3.4 and the papers [44] and [45]) because their angular parts $\sin \left(\pi \alpha s^{-1}\left(\varphi-\varphi_{0}(s)\right)\right)$ and $\sin \left(2 \pi \alpha s^{-1}\left(\varphi-\varphi_{0}(s)\right)\right)$ are orthogonal in the space $L_{2}\left(\varphi_{0}(s), \varphi_{0}(s)+\alpha(s)\right)$. 
Remark 3.4. Let $s_{0} \in \Gamma$ be a point where the convexity property of $\Omega$ is changed, i.e., $\alpha\left(s_{0}\right)=\pi$. Let, for definiteness, $\pm\left(\alpha\left(s_{0} \pm t\right)-\pi\right)>0$ for small $t \in\left(0, t_{0}\right)$. In this case, there exist two power-law solution families:

$$
\left\{U^{1,1+}(\cdot, s)\right\}_{s \in\left(s_{0}-t_{0}, s_{0}+t_{0}\right)} \quad \text { and } \quad\left\{U^{1,1-}(\cdot, s)\right\}_{s \in\left(s_{0}-t_{0}, s_{0}+t_{0}\right)},
$$

for which the graphs of homogeneity exponents,

$$
\Gamma \ni s \mapsto \pi / \alpha(s) \quad \text { and } \quad \Gamma \ni s \mapsto 2-\pi / \alpha(s),
$$

intersect at the point $s_{0}$. Having the same angular parts, the power-law solutions (3.32) do interact in the following sense. At $s=s_{0}$, equality (3.21) is valid, and the model problem on the half-plane $\mathbb{K}\left(s_{0}\right)=\{y: r>$ $\left.0, \varphi \in\left(\varphi_{0}\left(s_{0}\right), \varphi_{0}\left(s_{0}\right)+\pi\right)\right\}$ gets solution $(3.22)$ that depends on $\log r$ linearly. We mention here the papers [44] and [45], where these effects are discussed for much more general, though somewhat different situations (cf. Sec. 4.3).

3.5. Positivity preserving property for the biharmonic boundary-value problem. In the case of a locally convex domain $\Omega$, the generalized solutions $u$ and $v$ of problems (1.3), (1.4), and (1.5) coincide, and, as was explained in Sec. 1.4, these solutions are positive for a nonnegative right-hand side $f \in L_{2}(\Omega) \backslash\{0\}$. To examine solutions in a locally concave domain, we need the following assertion.

Lemma 3.1. A function $z \in V_{\beta}^{2}(\Omega)$ satisfies the following relation:

$$
\rho(x)^{\beta(x)-1 / 2}|z(x)| \leq c\left\|z ; V_{\beta}^{2}(\Omega)\right\|, \quad x \in \Omega .
$$

Proof. Due to the Sobolev imbedding theorem for $W_{2}^{2}(\Omega) \subset C(\bar{\Omega})$, it is sufficient to consider a function $z$ on the set $\Omega \cap \mathcal{U}_{d}$ introduced in Sec. 2.3. Let $\Xi_{1, k}^{j, q}$ be one of bricks (2.18). Performing the coordinate changes $x \mapsto \xi \mapsto \eta$ (see (2.13) and (2.19)) and recalling the definition of norm (2.1), we find out that the function $\widehat{\Xi}_{1, k}^{j, q} \ni \eta \mapsto Z(\eta)=z(x)$ satisfies the following chain of inequalities:

$$
\begin{aligned}
\left\|z ; V_{\beta}^{2}(\Omega)\right\|^{2} & \geq c\left(\sum_{p=0}^{2}\left\|r^{\beta-2+p} \nabla_{\xi}^{p} z ; L_{2}\left(\Xi_{1, k}^{j, q}\right)\right\|^{2}\right)^{1 / 2} \geq c\left(\sum_{p=0}^{2} 2^{-k\left(\beta\left(\xi^{j, q, k}\right)-1 / 2\right)} 2^{2 k p} 2^{-3 k}\left\|\nabla_{\xi}^{p} Z ; L_{2}\left(\widetilde{\Xi}_{1, k}^{j, q}\right)\right\|^{2}\right)^{1 / 2} \\
& \geq c 2^{-k\left(\beta\left(\xi^{j, q, k}\right)-1 / 2\right)}\left\|Z ; W_{2}^{2}\left(\widehat{\Xi}_{1, k}^{j, q}\right)\right\| \geq c 2^{-k\left(\beta\left(\xi^{j, q, k}\right)-1 / 2\right)} \sup _{\eta \in \widehat{\Xi}_{1, k}^{j, q}}|Z(\eta)| \geq c \sup _{\xi \in \Xi_{1, k}^{j, q}}|z(x)| .
\end{aligned}
$$

Here we have used the above-mentioned Sobolev theorem on a set $\widehat{\Xi}_{1, k}^{j, q}$ of unit size.

Let us show that the generalized solution $u \in W_{2,0}^{2}(\Omega)$ of problem (1.3) can lose the positivity property (1.14).

Proposition 3.1. Assume that condition (1.12) and condition (1.15) for a point $s_{0} \in \Gamma$ are satisfied. Then there exists a nonnegative function $f \in L_{2}(\Omega)$ for which the solution $u \in W_{2,0}^{2}(\Omega)$ of problem (1.3) changes sign inside the domain $\Omega$.

Proof. Fix indices $l=2$ and $\gamma$ so that requirements (3.16) and (3.27) are fulfilled and

$$
\gamma(s)=\frac{5}{2}-\frac{2 \pi}{\alpha(s)}-2 \varepsilon, \quad s \in \Upsilon_{0}:=\left[s_{0}-t_{0}, s_{0}+t_{0}\right],
$$

for small $\varepsilon>0$ and $t_{0}>0$, where

$$
3-\gamma(s)>\frac{2 \pi}{\alpha(s)}+\frac{1}{2}-\frac{2 \pi}{\alpha(s)}>2-\frac{\pi}{\alpha(s)}+\frac{1}{2}, \quad s \in \Upsilon_{0}
$$

(see Fig. 1 and cf. condition $(3.27)_{2}$ with $l=2$ ). This choice of index $\gamma$ is possible because

$$
\frac{2 \pi}{\alpha(s)}+\frac{1}{2}<\frac{3 \pi}{\alpha(s)} \quad \text { for } \quad \alpha(s) \in\left(\frac{3}{2} \pi, 2 \pi\right) .
$$


Now fix index $\sigma$ so that

$$
0<\sigma(s)<2 \text {, }
$$

in addition to requirements (3.16) and (3.27) . Condition (3.34) is much more restrictive than (3.16). It is easy to observe that due to our choice $(3.24)$, the intersection of the intervals $(0,2)$ and $(\gamma(s), \gamma(s)+1)$ is not empty, i.e., one can fulfill inequalities (3.34). Then $f \in L_{2}(\Omega) \subset V_{\sigma}^{0}(\Omega)$, and solutions of problem (1.3) in the spaces $W_{2,0}^{2}(\Omega)$ and $V_{\sigma}^{4}(\Omega) \subset W_{2}^{2}(\Omega)$ given by Theorems 3.2 and 3.3, respectively, coincide.

Consider the sets $G_{i}=\left\{x \in \Omega \cap \mathcal{U}_{d i / 2}:\left|s-s_{0}\right|<t_{0} i / 2\right\}, i=1,2$, and a cut-off function $\chi \in C^{\infty}(\bar{\Omega})$ that is equal to 1 on $G_{1}$ and 0 outside $G_{2}$. The product $u^{0}=\chi_{0} u$ satisfies problem (3.10) with right-hand sides

$$
f^{0}=\chi f+\left[\Delta_{x}^{2}, \chi\right] u \in V_{\gamma}^{0}(\Omega), \quad g_{1}^{0}=\left[\Delta_{x}, \chi\right] u \in V_{\gamma}^{3 / 2}(\Omega), \quad g_{0}^{0}=0 .
$$

The inclusions into the spaces with weight index $\gamma$ are true due to the following reasons: first, $\gamma(s)>0$ for $s \in \Upsilon_{0}$ due to equality (3.33), i.e., $f \in L_{2}\left(G_{2}\right) \subset V_{\gamma}^{0}\left(G_{2}\right)$, and, second, the commutators $\left[\Delta_{x}^{2}, \chi\right]$ and $\left[\Delta_{x}, \chi\right]$ are differential operators of the first and third order, respectively, with smooth coefficients; therefore,

$$
\left[\Delta_{x}^{2}, \chi\right] u \in V_{\sigma}^{1}(\Omega) \subset V_{\sigma}^{0}(\Omega) \quad \text { and } \quad\left[\Delta_{x}, \chi\right] u \in V_{\sigma}^{3}(\Omega) \subset V_{\gamma}^{2}(\Omega)
$$

by formula $(3.27)_{1}$. The hypotheses of Theorem 3.4 are satisfied, and hence the function $u^{0}$ admits representation (3.38) with intensity factors $K_{i}^{0} \in H^{\varkappa_{i}(\cdot)}(\Gamma), i=1,2$, and remainder $\tilde{u}^{0} \in V_{\gamma}^{4}(\Omega) \subset V_{\gamma-2}^{2}(\Omega)$. By Lemma 3.1 and relation (3.33),

$$
\left|\widetilde{u}^{0}(x)\right| \leq c r^{-(\gamma(s)-2)+1 / 2}\left\|\widetilde{u}^{0} ; V_{\gamma-2}^{2}(\Omega)\right\| \leq c r^{2 \varepsilon+2 \pi / \alpha(s)} \mathbf{n},
$$

where $\mathbf{n}$ is the sum of functions (3.35), which does not exceed $c\left\|f ; L_{2}(\Omega)\right\|$. Formulas (3.29) and (3.33) show that

$$
1>\varkappa_{2}(s)=3-\gamma(s)-\frac{2 \pi}{\alpha(s)}=2 \varepsilon+\frac{1}{2} \quad \text { and } \quad 0<\varkappa_{1}(s)=2 \varepsilon+3\left(\frac{\pi}{\alpha(s)}-\frac{1}{2}\right)<\frac{1}{2} .
$$

Reducing the value $t_{0}>0$, if necessary, we may assume that

$$
\varkappa_{0} \leq \varkappa_{1}(s) \leq \varkappa_{0}+\varepsilon, \quad s \in \Upsilon_{0}
$$

Thus,

$$
K_{2}^{0} \in H^{2 \varepsilon+1 / 2}(\Gamma) \subset C^{0,2 \varepsilon}(\Gamma)
$$

and

$$
K_{1}^{0} \in H^{\varkappa_{1}^{(\cdot)}}(\Gamma) \subset H^{\varkappa_{0}(\cdot)}(\Gamma) \subset L_{q}(\Gamma), \quad q=\left(\frac{1}{2}-\varkappa_{0}\right)^{-1} \in(2+\infty) .
$$

We may assume that in definition (2.35) of the extension operator $\mathfrak{C}$, one of the functions in the family $\left\{\chi_{\Gamma}^{i}\right\}$, say, $\chi_{\Gamma}^{i_{0}}$, equals one on the arc $\Upsilon_{0}$, and

$$
\mathfrak{C}\left(K_{ \pm}^{0} ; r, s\right)=\chi_{\Omega}^{i_{0}}(r, s) \int_{\mathbb{R}} X(t) K_{ \pm}^{0}(s-r t) d t
$$

Applying inclusions (3.38) and formulas (3.20), (3.37), (3.29), and (2.34) and taking into account the compactness of supports of intensity factors, we deduce that

$$
\begin{gathered}
\left|\mathfrak{C}\left(K_{1}^{0} ; r, s\right) U^{1,1-}(y, s)\right| \leq c r^{2-\pi / \alpha(s)}\left\|X ; L_{q /(q-1)}(\mathbb{R})\right\|\left(\int_{\mathbb{R}} K_{1}^{0}(s-r t)^{q} d t\right)^{-1 / q} \\
\leq c r^{2-\pi / \alpha(s)} r^{1 / q}\left\|K_{1}^{0} ; L_{q}(\mathbb{R})\right\| \leq c r^{2-\pi / \alpha(s)} r^{\varkappa_{0}-1 / 2} \mathbf{n} \leq c r^{\varepsilon+2 \pi / \alpha(s)} \mathbf{n}, \\
\left|\left(K_{2}^{0} ; r, s\right) U^{2,0+}(y, s)-K_{2}^{0}(s) U^{2,0+}(y, s)\right| \leq c r^{2 \pi / \alpha(s)}\left|\int_{\mathbb{R}} X(t)\left(K_{2}^{0}(s-r t)-K_{2}^{0}(s)\right) d t\right|
\end{gathered}
$$




$$
\begin{gathered}
\leq c r^{2 \pi / \alpha(s)} r^{2 \varepsilon} \int_{\mathbb{R}}|X(t)|\left(t^{2}\right)^{\varepsilon} d t \sup _{h \in \mathbb{R}} h^{-2 \varepsilon}\left|K_{2}^{0}(s-h)-K_{2}^{0}(s)\right| \\
\leq c r^{2 \varepsilon+2 \pi / \alpha(s)}\left\|K_{2}^{0} ; C^{0,2 \varepsilon}(\mathbb{R})\right\| \leq c r^{2 \varepsilon+2 \pi / \alpha(s)} \mathbf{n} .
\end{gathered}
$$

Thus, due to relations (3.36) and (3.39), decomposition (3.28) of the function $u^{0}=\chi_{0} u$ on the set $G_{2}$ takes the following form:

$$
u^{0}(x)=K_{2}^{0}(s) r^{2 \pi / \alpha(s)} \sin \frac{2 \pi}{\alpha(s)}\left(\varphi-\varphi_{0}(s)\right)+O\left(r^{\varepsilon+2 \pi / \alpha(s)} \mathbf{n}\right) .
$$

The sine in the right-hand side of (3.40) has different signs for $\pm\left(\varphi-\varphi_{0}(s)\right)>0$. Hence, it suffices to verify that there exists a nonnegative function $f \in L_{2}(\Omega) \backslash\{0\}$ for which the intensity factor $K_{2}^{0}(s)=\chi_{0}(x) K_{2}(s)$ is not identically zero on the arc $\Upsilon_{0}$.

Let $H \in C_{c}^{\infty}\left(s_{0}-t_{0} / 2, s_{0}-t_{0} / 2\right)$. Similarly to formula (2.38), we search for a solution of the homogeneous problem (1.3) in the form

$$
\zeta^{b i}(H ; x)=\chi(r) H(s) U^{2,1-}(y, s)+\widehat{\zeta}^{b i}(H ; x),
$$

where $\widehat{\zeta}^{b i}(H ; \cdot) \in V_{\sigma}^{l+2}(\Omega)$ is a solution of problem $(3.10)$ with right-hand sides

$$
f=-\Delta_{x}^{2} \chi H U^{2,1-}, \quad g_{1}=-\Delta_{x} \chi H U^{2,1-}, \quad g_{0}=-\chi H U^{2,1-} .
$$

Since $U^{2,1-}$ is a power-law solution of the model problem (3.19), the same reasoning as in the beginning of Sec. 2.5 establishes inclusion (3.11) for any weight index $\sigma$ that meets the inequality

$$
\sigma(s)-l>2 \pi / \alpha(s), \quad s \in \Upsilon_{0}
$$

Note that the function $\chi H U^{2,1-}$ belongs to the space $V_{\beta}^{l+2}(\Omega)$ under a much more restrictive condition $\beta(s)-$ $l-1>2 \pi / \alpha(s)$. According to the inequalities $3 \pi / 2<\alpha(s)<2 \pi, s \in \Upsilon_{0}$, condition (3.16) on the arc $\Upsilon_{0}$ turns into the following one:

$$
1-2 \pi / \alpha(s)<\sigma(s)-l<1-2 \pi / \alpha(s) .
$$

Hence, for any $l \in \mathbb{N}_{2}$ there exists a function $\sigma \in C^{\infty}(\Gamma)$ subjected to both requirements (3.16) and (3.43). Now Theorem 3.3 gives us a solution $\widehat{\zeta}^{b i}(H ; \cdot) \in V_{\sigma}^{l+2}(\Omega)$ of problem (3.10) with right-hand side (3.42). Simultaneously, $\zeta^{b i}(H ; \cdot)$ becomes a nontrivial solution of the homogeneous problem (3.10) or (1.3). Results of [12] (see also [39, $\S 9.4])$ provide an analog of formula (2.26) for the intensity factor $K_{2}=K_{2}^{0}$ in decomposition (3.28) of the solution $u$ to problem $(1.3)$ on the set $G_{1}$ :

$$
\int_{\Omega} f(x) \zeta^{b i}(H ; x) d x=8 \pi \int_{\Gamma}\left(1-\frac{2 \pi}{\alpha(s)}\right) K_{2}(s) H(s) d s .
$$

Note that formula (3.44) differs slightly from formula (2.46) which is related to the Dirichlet problem for the Laplace operator. Indeed, the procedure described above for construction $\zeta^{i}(H ; \cdot)$ allows for singularities $H(s) U^{2,1-}(y, s)$ only on parts of the edge $\Gamma$ at which $\alpha(s) \in(3 \pi / 2,2 \pi)$. For an arbitrary opening angle, the structure of the weight function is far more complicated (see $[13,11]$ and $[39, \S 8.3]$ ). This statement matches with the fact that the asymptotic decompositions (3.28) and (3.40) were derived on the set $G_{1}$ only.

The singular part $\chi H U^{2,1-}$ and, therefore, the weight function (3.41) itself changes sign inside the domain $\Omega$. Hence, for a fixed nontrivial density $H \in C_{c}^{\infty}\left(s_{0}-t_{0} / 2, s_{0}+t_{2} / 2\right)$, the integral over the domain $\Omega$ in formula (3.44) can be supplied with any sign by a proper choice of the right-hand side $f \in L_{2}(\Omega) \backslash\{0\}$ of problem (1.3). The corresponding intensity factor $K_{+}$cannot vanish everywhere on the arc $\Upsilon_{0}$.

\section{Body WITH A CRACK AND OTHER GEOMETRICAL SHAPES}

4.1. Edge with opening angle $2 \pi$. The case $\alpha(s)=2 \pi$ (the edge is the front of a crack) was excluded from consideration in Sec. 3 by condition (1.12). This restriction was essential since the operator of problem (3.10) in the Kondrat'ev spaces is never Fredholm (see Remark 3.1). An indirect reason due to which the scale of spaces $V_{\beta}^{l}((\Omega)$ is unsuitable is the appearance of two types (3.21) and (3.22) of power-logarithmic solutions which cannot be distinguished in the scale, and also the collapse of the interval $I(2 \pi)$ (see Remark 3.2, in particular, formula 
(3.25), and also Fig. 1). A similar obstacle appears in the simplest Neumann problem for the Poisson equation in a domain $\Omega \subset \mathbb{R}^{3}$ with a smooth one-dimensional edge of arbitrary opening; namely, the model Neumann problem (we change the boundary condition in (2.25)) has the solutions $U^{0}(y)=1$ and $U^{1 / 2}(y)=\log r$, while the functions $x \mapsto \chi(r) H_{0}(s)$ and $x \mapsto \chi(r) H_{1 / 2}(s) \log r$ belong or do not belong to the space $V_{\beta}^{l+1}(\Omega)$ simultaneously (for $\beta(s)>0$ and $\beta(s)<0$, respectively).

In $[14,15]$, the so-called scale of step-weighted spaces $V_{\beta}^{l, h}(\Omega)$ was introduced; in these spaces, norms with $l, h \in \mathbb{N}_{0}, h<l$, and $\beta \in C^{\infty}(\Gamma)$ take the following form:

$$
\left\|U ; V_{\beta}^{l, h}(\Omega)\right\|=\left(\sum_{k=0}^{l}\left\|\rho^{\beta-l+k+\Theta(h-k)} \nabla_{x}^{k} U ; L_{2}(\Omega)\right\|^{2}\right)^{1 / 2},
$$

where $\Theta$ is the Heaviside function, $\Theta(t)=1$ for $t \geq 0$ and $\Theta(t)=0$ for $t<0$, while the remaining notation is the same as in formula (2.1). Within this scale, the power-logarithmic solutions (3.21) and (3.22) can be distinguished. For a density $H \in C^{\infty}(\Gamma)$, the function $x \mapsto \chi(r) H(s) U^{2,0+}(y, s)$ belongs to the space $V_{\sigma}^{l+2,1}(\Omega)$ with $l \in \mathbb{N}_{2}$ and $\sigma(s)-l \in(-1,0)$, while the function $x \mapsto \chi(r) H(s) U^{2,1 / 2}(y, s)$ does not belong to the above space since the first function is smooth, but derivatives of order $k>1$ of the second function have singularities $O\left(r^{1-k}\right)$.

The operator

$$
\mathcal{N}_{\beta}^{l, 0}=\left\{-\Delta_{x},\left.\partial_{n}\right|_{\partial \Omega \backslash \Gamma}\right\}: V_{\beta}^{l+1,0}(\Omega) \rightarrow V_{\beta}^{l-1}(\Omega) \times V_{\beta}^{l-1 / 2}(\partial \Omega)
$$

of the above-mentioned Neumann problem for the Poisson equation becomes Fredholm of index zero if $l \in \mathbb{N}$ and $\beta(s)-l \in(-1 / 2,0)$. We associate problem (1.2) with a mapping

$$
\mathcal{B}_{\beta}^{l, 1}: V_{\beta, 0, \Delta}^{l+2,1}(\Omega) \rightarrow V_{\beta}^{l-2}(\Omega)
$$

where $V_{\beta, 0, \Delta}^{l+2,1}(\Omega)$ is the subspace $\left\{u \in V_{\beta}^{l+2,1}(\Omega): u=0, \Delta_{x} u=0\right.$ on $\left.\partial \Omega \backslash \Gamma\right\}$.

Proposition 4.1. Assume that $\alpha(s)=2 \pi$ for $s \in \Gamma$ and $l \in \mathbb{N}_{2}$. Operator (4.2) of problem (1.2) is an isomorphism if and only if $\beta(s)-l \in(-1 / 2,0)$ for $s \in \Gamma$. If the inclusion fails at least at a single point on the edge, the operator loses the Fredholm property.

Proof. A proof of the first assertion can be performed along the same lines as in [15] (see also [39, §6.4]). If the inequalities $\beta-l>0$ or $\beta-l<-1 / 2$ are met on an open nonempty arc, then the operator in (4.2) gets an infinite-dimensional kernel or co-kernel, respectively (see $[9,11])$. If $\beta\left(s_{0}\right)-l=0$ or $\beta\left(s_{0}\right)-l=-1 / 2$ at a single point on the edge, then the lost of the Fredholm property can be verified in the same way as in Remark 2.3.

4.2. Asymptotics of the solution near the crack front. In this section, we take $l=2$ and $\beta \in(3 / 2,2)$. If $f \in L_{2}(\Omega) \subset V_{\beta}^{0}(\Omega)$, then, by Proposition 4.1, there exists a unique solution $u \in V_{\beta, 0, \Delta}^{4}(\Omega)$, and the estimate

$$
\left\|u ; V_{\beta, 0, \Delta}^{4}(\Omega)\right\| \leq c\left\|f ; V_{\beta}^{0}(\Omega)\right\|
$$

is valid. Due to the inequality $\beta<2$ and definition of the norm in (4.1), the space $V_{\beta, 0, \Delta}^{4}(\Omega)$ is contained in $W_{2,0}^{2}(\Omega)$, i.e., $u$ is a generalized solution of problem (1.3), and relation (4.4) provides estimate (2.9). The asymptotic formula in the next assertion contains a term $\mathfrak{C}\left(K_{2}\right) U^{2,0+}$ that changes sign inside the domain $\Omega$. This extends Proposition 3.1 to a domain with a crack.

Proposition 4.2. Assume that $\alpha(s)=2 \pi$ for $s \in \Gamma, l=2, \beta \in(3 / 2,2), \gamma \in(1,3 / 2)$, and $f \in V_{\beta}^{0}(\Omega)$. Then the solution $u \in V_{\beta, 0, \Delta}^{4,1}(\Omega)$ of problem (1.3) guaranteed by Proposition 4.1 admits the asymptotic form

$$
u(x)=\mathfrak{C}\left(K_{2} ; r, s\right) U^{2,0+}(y, s)+\mathfrak{C}\left(K_{1} ; r, s\right) U^{1,1-}(y, s)++\mathfrak{C}\left(K_{3} ; r, s\right) U^{3,0+}(y, s)+\widetilde{u}(x),
$$

where $K_{2} \in H^{2-\gamma}(\Gamma), K_{1}, K_{3} \in H^{-\gamma+3 / 2}(\Gamma)$, and $\tilde{u} \in V_{\gamma}^{4}(\Omega)$, while the norms of these functions do not exceed the value $c\left\|f ; V_{\gamma}^{0}(\Omega)\right\|$.

Proof. Due to Lemma 6.4 .8 of $[6]$, the function $u \in V_{\sigma, 0, \Delta}^{4,1}(\Omega)$ has a decomposition

$$
u(x)=\mathfrak{C}\left(K_{2} ; r, s\right) U^{2,0+}(y, s)+\widehat{u}(x),
$$


where $\hat{u} \in V_{\sigma}^{4}(\Omega)$ and $K_{2} \in H^{2-\sigma}(\Gamma)$. Repeating (with slight modifications) a procedure from [37, 38, 11, 13] (see also [39, Chapter 9]) for derivation of asymptotics for solutions to elliptic boundary-value problems near smooth edges, which includes local rectification of the edge $\Gamma$ and the surfaces $\partial \Omega_{ \pm}$and extraction of principal parts of differential operators, we obtain decomposition (4.4), and, in particular, we improve representation (4.5) of the differential properties of the factor $K_{2}$ and increase the decay rate of the asymptotic remainder.

The proof of existence of a nonnegative right-hand side $f \in L_{2}(\Omega)$ for which the solution $u$ changes sign inside $\Omega$ follows in general the scheme of Sec. 3.4. We observe only one distinction: the weight function $\zeta^{\text {bi }}(H ; \cdot)$, which figures in the integral representation (3.44) of the intensity factor $K_{2}$, is generated by the power-logarithmic solution (3.22). The multiplier $\log r$ makes the construction of the weight function much more cumbersome, and we skip it here (see the paper [13] and also [39, §9.3]) for the general situation).

4.3. Cases $\alpha\left(s_{0}\right)=2 \pi$ and $\alpha\left(s_{0}\right)=\pi$. If the edge becomes a crack at one point or on an arc, but $\alpha(s)<2 \pi$ on the remaining part of the contour $\Gamma$, then the operator of problem (3.10) cannot be Fredholm in both scales of the weighted spaces $V_{\sigma}^{l+2}(\Omega)$ and $V_{\beta}^{l+2,1}(\Omega)$. The Kondrat'ev classes do not fit due to Remark 3.1, and step-weighted spaces do not fit since under the condition $\beta-l \in(-1 / 2,0)$ (Proposition 4.2), the product $\chi H U^{2,0+}$ does not belong to the space $V_{\beta}^{l+2,1}(\Omega)$ in the case $\alpha(s)<2 \pi$. An analogous obstacle appears if the convexity property of the domain $\Omega$ is changed along the edge: the model problem (3.19) in the half-plane $\mathbb{K}(\pi)=\mathbb{R}_{+}^{2}$ has the same power-logarithmic solutions (3.21) and (3.22).

A point $s_{0} \in \Gamma$ where $\alpha\left(s_{0}\right)=2 \pi$ or $\alpha\left(s_{0}\right)=\pi$ can be interpreted as the top of a polyhedral angle (cf. [46] and also [39, Chapter 7]). However, the complete theory of such boundary singularities has not been created yet, and the known particular results do not cover the questions under discussion.

Under restriction (1.2), which permits $\alpha\left(s_{0}\right)=2 \pi$, or in the cases $\alpha\left(s_{0}\right)=\pi$ and $\pm(\alpha(s)-\pi) \geq 0$, a formal decomposition of the solution $u \in W_{2,0}^{2}(\Omega)$ of problem (1.3) does not differ from those in Theorem 3.4 and Proposition 4.2 since the power-law solutions (3.20), which must be put into the asymptotics, depend smoothly on parameter $s$. If the convexity property of the domain $\Omega$ is changed at the point $s_{0}$, then, according to results of Secs. 3.2 and 3.4 near "convex" and "concave" parts of the edge $\Gamma$, the asymptotic decomposition must contain the terms $K_{1+}(s) U^{1,0+}(y, s)$ and $K_{1-}(s) U^{1,1-}(y, s)$, respectively. The angular parts of these powerlaw solutions match smoothly but the radial parts $r^{\pi / \alpha(s)}$ and $r^{2-\pi / \alpha(s)}$ match only continuously. Hence, the structure of asymptotic decomposition of the generalized solution of problem (1.3) (if such a solution exists) has to be changed compared to decompositions (3.28) or (4.4). The authors do not know results on such nonsmooth asymptotic expansions near a smooth edge. We emphasize that the papers [44] and [45] deal with lost of smoothness of a different type.

This research is supported by the Netherlands Organization for Scientific Research (NWO) and the RFBR (project 047.017.020).

Translated by S. A. Nazarov.

\section{REFERENCES}

1. A. D. Aleksandrov and N. Yu. Netsvetaev, Geometry [in Russian], Moscow (1990).

2. M. Sh. Birman, "Variational methods of solution of boundary-value problems analogous to the method of Trefftz," Vestn. Leningr. Univ., 11, 69-89 (1956).

3. M. Sh. Birman and G. E. Skvortsov, "Square summability of highest derivatives of a solution of the Dirichlet problem in a domain with piecewise smooth boundary," Izv. VUZ., 30, 11-21 (1962).

4. T. Boggio, "Sull'equilibrio delle piastre elastiche incastrate," Rend. Ac. Lincei., 10, 197-205 (1901).

5. T. Boggio, "Sulle funzioni di Green d'ordine m," Rend. Circ. Mat. Palermo, 20, 97-135 (1905).

6. B. M. Brown, E. B. Davies, P. K. Jimack, and M. D. Mihajlović, "A numerical investigation of the solution of a class of fourth-order eigenvalue problems," R. Soc. Lond. Proc., Ser. A, Math. Phys. Eng. Sci., 456, 1505-1521 (2000). .

7. C. V. Coffman and R. J. Duffin, "On the structure of biharmonic functions satisfying the clamped plate conditions on a right angle," Adv. Appl. Math., 1, 373-389 (1980).

8. C. V. Coffman, "On the structure of solutions to $\Delta^{2} u=\lambda u$ which satisfy the clamped plate condition on a right angle," SIAM J. Math. Anal., 13, 746-757 (1982).

9. M. Costabel and M. Dauge, "Stable asymptotics for elliptic systems on plane domains with corners," Comm. Partial Diff. Eqs., 19, 1677-1726 (1994). 
10. A. Dall'Acqua and G. Sweers, "The clamped plate equation for the Limaçon," Annali Matematica, 184, 361-374 (2005).

11. R. J. Duffin, "On a question of Hadamard concerning super-biharmonic functions," J. Math. Phys., 27, 253-258 (1949).

12. P. R. Garabedian, "A partial differential equation arising in conformal mapping," Pacific J. Math., 1, 485-524 (1951).

13. H.-C. Grunau and G. Sweers, "Positivity for perturbations of polyharmonic operators with Dirichlet boundary conditions in two dimensions," Math. Nachr., 179, 89-102 (1996).

14. H.-C. Grunau and G. Sweers, "Positivity for equations involving polyharmonic elliptic operators with Dirichlet boundary conditions," Math. Ann., 307, 589-626 (1997).

15. J. Hadamard, "Sur certains cas intéressants du problème biharmonique," in: Atti IV Congr. Intern. Mat. Rome (1909), pp. 12-14.

16. J. Hadamard, "Mémoire sur le problème d'analyse relatif à l'équilibre des plaques élastiques incastrées," Mémoires présentés par divers savants a l'Académie des Sciences, 33, 1-128 (1908).

17. V. A. Kondrat'ev, "Smoothness of a solution of the Dirichlet problem for second-order elliptic equations in a piecewise smooth domain," Differents. Uravn., 6, 1831-1843 (1970).

18. V. A. Kondrat'ev, "Singularities of a solution of the Dirichlet problem for a second-order elliptic equation in a neighborhood of an edge," Differents. Uravn., 13, 2026-2032, 2109 (1977);

V. A. Kozlov, V. A. Kondrat'ev, and V. G. Maz'ya, "On sign variation and the absence of 'strong' zeros of solutions of elliptic equations," Izv. Akad Nauk SSSR, 34, 337-353 (1990).

19. V. A. Kozlov, V. G. Maz'ya, and J. Rossmann, Elliptic Boundary-Value Problems in Domains with Point Singularities, Amer. Math. Soc., Providence, RI (1997).

20. O. A. Ladyzhenskaya, The Boundary-Value Problems of Mathematical Physics [in Russian], Moscow (1973).

21. C. Loewner, "On generation of solutions of the biharmonic equation in the plane by conformal mappings," Pacific J. Math., 3, 417-436 (1953).

22. V. G. Maz'ya and B. A. Plamenevskii, "Elliptic boundary-value problems in a domain with a piecewise smooth boundary," in: Proceedings of the Symposium on Continuum Mechanics and Related Problems of Analysis, Vol. 1 (Tbilisi, 1971), Tbilisi (1973), pp. 171-181.

23. V. G. Maz'ya and B. A. Plamenevskii, "Coefficients in asymptotic expansions of solutions of elliptic boundaryvalue problems near an edge," Dokl. Akad. Nauk SSSR, 229, 33-36 (1976).

24. V. G. Maz'ya and B. A. Plamenevskii, "Elliptic boundary-value problems on manifolds with singularities," in: Probl. Mat. Anal., No. 6, Leningrad. Univ. (1977), pp. 85-142.

25. V. G. Maz'ya and B. A. Plamenevskii, "The coefficients in the asymptotics of solutions of elliptic boundaryvalue problems with conical points," Math. Nachr., 76, 29-60 (1977).

26. V. G. Maz'ya, S. A. Nazarov, and B. A. Plamenevskii, "Bending of an almost polygonal plate with freely supported boundary," Izv. VUZ., 8, 34-40; 27, 40-48 (1983).

27. V. G. Maz'ya and J. Rossmann, "Über die Lösbarkeit und die Asymptotik der Lösungen elliptischer Randwertaufgaben in Gebieten mit Kanten," Preprint 31/84 Karl-Weierstrass-Inst. Math., Berlin (1984).

28. V. G. Maz'ya and J. Rossmann, "Über die Asymptotik der Lösungen elliptischer Randwertaufgaben in der Umgebung von Kanten," Math. Nachr., 138, 27-53 (1988).

29. V. G. Mazja, S. A. Nazarov, and B. A. Plamenewskii, Asymptotische Theorie Elliptischer Randwertaufgaben in Singulär Gestörten Gebieten, 1, Akademie-Verlag, Berlin (1991).

30. V. G. Maz'ya and J. Rossmann, "Stable asymptotics of solutions to the Dirichlet problem for elliptic equations of second order in domains with angular points or edges," Operator Theory, Advances, Appl., 57, 215-224 (1992).

31. S. G. Mikhlin, Variational Methods in Mathematical Physics, Translated by T. Boddington; editorial introduction by L. I. G. Chambers, Pergamon Press, New York (1964).

32. S. A. Nazarov, "Parameter asymptotics of a solution to an elliptic boundary-value problem with periodic coefficients in a cylinder," Differents. Uravn. Primen., 30, 27-46 (1981).

33. S. A. Nazarov, "Estimates near the edge of a solution of the Neumann problem for an elliptic system," Vestn. Leningr. Univ., 1, 37-42 (1988).

34. S. A. Nazarov, "Derivation of the variational inequality for small increase of mode-one crack," Mekh. Tverd. Tela, 2, 152-160 (1989).

35. S. A. Nazarov and B. A. Plamenevskii, "Neumann problem for self-adjoint elliptic systems in a domain with piecewise smooth boundary," Trudy Leningr. Mat. Ob., 1, 174-211 (1990). 
36. S. A. Nazarov and B. A. Plamenevskii, "Self-adjoint elliptic problems with radiation conditions on edges of the boundary," Algebra Analiz, 4, 196-225 (1992).

37. S. A. Nazarov and B. A. Plamenevskii, "Elliptic problems with radiation conditions on edges of the boundary," Mat. Sb., 183, 13-44 (1992).

38. S. A. Nazarov and B. A. Plamenevskii, "A generalized Green's formula for elliptic problems in domains with edges," in: Probl. Mat. Anal., 13, St.-Petersburg Univ. (1992), pp. 106-147.

39. S. A. Nazarov and B. A. Plamenevskii, Elliptic Problems in Domains with Piecewise Smooth Boundaries, Walter de Gruyter, Berlin-New York (1994).

40. V. A. Nikishkin, "Singularities of a solution of the Dirichlet problem for a second-order equation in a neighborhood of an edge," Vestn. Moskov. Univ., Ser. I, Mat. Mekh., No. 2, 51-62, 103 (1979).

41. S. Osher, "On Green's function for the biharmonic equation in a right angle wedge," J. Math. Anal. Appl., 43, 705-716 (1973).

42. O. M. Sapongyan, Bending of Thin Elastic Plates [in Russian], Aiastan, Erevan (1975).

43. H. S. Shapiro and M. Tegmark, "An elementary proof that the biharmonic Green function of an eccentric ellipse changes sign," SIAM Rev., 36, 99-101 (1994).

44. G. Sweers, "When is the first eigenfunction for the clamped plate equation of fixed sign?" in: Electron. J. Diff. Eqns. Conf. (2001), pp. 285-296.

45. G. Szegö, "Remarks on the preceeding paper of Charles Loewner," Pacific J. Math., 3, 437-446 (1953).

46. H. Triebel, Interpolation Theory, Function Spaces, and Differential Operators, North-Holland Mathematical Library, 18, North-Holland, Amsterdam-New York (1978). 